\title{
M-learning: aceptación tecnológica de dispositivos móviles en la formación online
}

\section{Extracto:}

\section{Sumario}

1. Introducción

2. La aceptación tecnológica del m-learning

3. Metodología de investigación

4. Diseño de la investigación

5. Evaluación del modelo propuesto

6. Análisis de resultados

7. Conclusiones e implicaciones prácticas

8. Bibliografía
El aprendizaje es una herramienta fundamental en el desarrollo de la Agenda Digital para Europa. Los dispositivos móviles son una tecnología en crecimiento y con unos ratios de uso entre la población cada vez más elevados, lo que hace que el futuro de la educación deba incorporar estos dispositivos. El m-learning es una dimensión crítica para alcanzar la Estrategia Europea 2020 y para mejorar la competitividad de la sociedad europea. El propósito de esta investigación es analizar los retos de la integración de los dispositivos móviles desde la perspectiva de la aceptación tecnológica en las actividades de aprendizaje en la educación superior. Identificamos los aspectos clave para lograr un adecuado desarrollo estratégico e innovador del m-learning. La principal conclusión de la investigación es que el uso de los dispositivos móviles es aceptado y considerado útil por los estudiantes para realizar sus actividades académicas. También hemos encontrado que los conocimientos tecnológicos no representan un freno a su desarrollo, ya que los jóvenes universitarios dominan la tecnología móvil y no necesitan formación alguna para emplearla como herramienta educativa. Demostramos que la formación de usuarios y el soporte técnico no son aspectos clave para el desarrollo estratégico de proyectos de m-learning, pero sí es determinante la adaptación de contenidos al entorno móvil.

Palabras clave: aprendizaje móvil, estrategias de e-learning, modelo de aceptación de tecnología (TAM), tecnología móvil.
Fecha de entrada: 03-05-2017

Fecha de aceptación: 04-07-2017

Fecha de revisión: 30-01-2018 


\section{M-learning: technological acceptance of mobile devices in online learning}

\section{Abstract:}

Learning is a fundamental tool to develop the Digital Agenda for Europe. Mobile devices are a growing technology with a high penetration in markets, consequently, the future of education must incorporate mobile technologies. The m-learning is a critical dimension to achieve the European 2020 Strategy and improve the competitiveness of European society. Therefore, the purpose of this research is to analyses the acceptance of mobile technologies for learning activities in high education. We identify the key aspects to achieve an adequate strategic and innovative development of m-learning. The main conclusion of the research is that the use of mobile devices is accepted and considered useful by students to carry out their academic activities. We have also found that technological support is not a restriction, since young university students dominate mobile technology and do not require any support to use it as an educational tool. We demonstrate that user training and technical support are not key aspects for the strategic development of m-learning projects, but whether the adaptation of contents to the mobile environment is crucial.

Keywords: mobile learning, e-learning strategies, technology acceptance model (TAM), mobile technology.

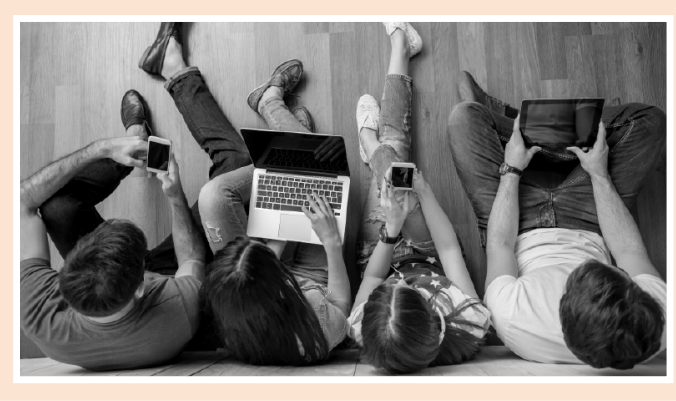

\section{INTRODUCCIÓN}

La generación de conocimiento es una estrategia fundamental para lograr los objetivos de la Estrategia Europea 2020. En este sentido, una parte fundamental de la Agenda Digital para Europa es incrementar los beneficios de las tecnologías de la información y la comunicación (TIC) en la sociedad actual. Las tecnologías móviles se han convertido en una herramienta esencial para el desarrollo de una comunidad europea digital, ya que su penetración ha crecido exponencialmente en la última década. De acuerdo con el informe La Sociedad de la Información en España 2016 (estudio elaborado por la Fundación Telefónica), las ventas de smartphones en España alcanzaron los 334,9 millones en el primer trimestre de 2016. Estas cifras representan el $87 \%$ (dato extraído del Informe Ditrendia 2016: Mobile en España y en el Mundo) del total de teléfonos móviles del país. En cuanto a la conectividad, el $92 \%$ de los internautas acceden a internet desde el smartphone. Por lo tanto, es un dispositivo que puede tener implicaciones importantes en diversas áreas de estudio, como, por ejemplo, en la educación.

Por otro lado, el uso de dispositivos móviles ha tenido un impacto en muchas de nuestras actividades diarias: comunicación, banca, compras, trabajo, etc., transformando nuestros comportamientos y hábitos. Govindarajan (2012) señala que algunos países desarrollados han invertido en la construcción de infraestructuras de líneas móviles con el fin de aumentar el alcance de los servicios ofrecidos a la población $(24 / 7 / 365)^{3}$, como la banca móvil o las compras a través de internet. El aprendizaje es una de las aplicaciones innovadoras en el emergente panorama de movilidad. El aprendizaje es una dimensión crítica de

\footnotetext{
${ }^{3}$ El concepto $24 / 7 / 365$ se usa para indicar que las TIC pueden ser utilizadas las 24 horas del día, los 7 días de la semana y los 356 días del año. Frente a otro tipo de servicios de información, las tecnologías no tienen restricción alguna: pueden ser empleadas de lunes a domingo, de 00:00 h a 23:59 h; y, en el caso de los dispositivos móviles, también desde cualquier lugar que tenga conexión a internet.
} 
la sociedad de la información y el conocimiento. Los dispositivos móviles facilitan el acceso a ese conocimiento de una manera inimaginable. Las tecnologías móviles presentan importantes vacíos de conocimiento que requieren ser investigados con rigor, y el comportamiento de la sociedad hacia el uso de los dispositivos móviles es uno de los más importantes. La innovación en el área móvil debe tener en cuenta lo que los usuarios de dispositivos móviles requieren para seguir consumiendo de alguna manera el conocimiento.

La velocidad de los cambios en la economía mundial obligó a las empresas a generar nuevas técnicas de formación y desarrollo de los individuos. Estas innovadoras técnicas son cada vez más eficaces mediante la integración de métodos de aprendizaje en línea y dispositivos móviles (m-learning). El aprendizaje en línea (en cualquiera de sus modalidades) tiene la ventaja de combinar la conectividad y el uso de la Web 2.0, es decir, está apuntando a la aplicación del paradigma de la ubicuidad: «En cualquier lugar y en cualquier momento». Este paradigma también tiene implicaciones importantes desde el punto de vista del negocio dentro de las instituciones educativas; por ejemplo, puede proporcionar una mayor competitividad en los mercados de aprendizaje, ofertando cursos, talleres y programas completos en línea. La gestión del conocimiento ayudaría a los ciudadanos y a las empresas a adquirir

\section{El propósito de esta investigación es analizar la aceptación de las tecnologías móviles para las actividades de aprendizaje como punto de partida para el desarrollo de una adecuada integración estratégica de estas herramientas en la educación superior}

conocimientos y a desarrollar mejores prácticas para incrementar su competitividad. El desarrollo de estrategias educativas nos permitirá construir una Europa mejor en las dimensiones sociales y económicas.

El propósito de esta investigación es analizar la aceptación de las tecnologías móviles para las actividades de aprendizaje como punto de partida para el desarrollo de una adecuada integración estratégica de estas herramientas en la educación superior. Con este análisis podemos identificar los aspectos clave que deben tener en cuenta las instituciones de educación superior (IES) en el desarrollo futuro de las estrategias de m-learning.

\section{LA ACEPTACIÓN TECNOLÓGICA DEL M-LEARNING}

Conocer el grado de aceptación de las tecnologías móviles es fundamental para lograr un adecuado empleo de estas en los procesos de aprendizaje. Los modelos de aceptación tecnológica permitirán a los responsables de la toma de decisiones y a los responsables de I+D comprender las razones para aceptar o rechazar una determinada tecnología, resultando este análisis muy útil tanto en el desarrollo como en la utilización de futuras innovaciones de software y hardware en el sector educativo.

Las IES deben considerar el panorama actual sobre el uso y la aceptación de la tecnología en la sociedad, es decir, han de tener en cuenta aspectos como los siguientes:

- ¿Cómo se mueve el mercado de las tecnologías?

- ¿Por qué los usuarios de las tecnologías eligen un dispositivo para ciertas actividades?

- ¿Cuál es el proceso de selección?
Sin lugar a dudas, estas preguntas ayudarán a tomar medidas en el sector educativo que mejoren su excelencia y eleven su competitividad, formulando estrategias de éxito que integren correctamente la tecnología adecuada, de la forma adecuada, en el momento adecuado. Estas acciones dependerán de la adopción de tecnología por parte de los usuarios. Entender las razones por las que un estudiante acepta usar su teléfono móvil en las actividades educativas será de gran valor para los directivos a la hora de tomar decisiones estratégicas. Por esta razón, consideramos importante el desarrollo y el análisis de un modelo empírico que explique la aceptación de las tecnologías dentro del sector educativo como parte previa de la innovación de producto.

\subsection{Modelo TAM}

Terzis y Economides (2011) señalan que uno de los recursos más utilizados para el estudio de la acepta- 
ción de tecnologías es el TAM. Este modelo fue desarrollado por Davis (1985) como una adaptación de la teoría de la acción razonada (TRA)4 (Fishbein y Ajzen, 1975), que proporciona información sobre el comportamiento de los seres humanos y permite determinar la dirección que hay que seguir en términos de creación o innovación tecnológica. Como podemos ver, la figura 1 muestra las variables fundamentales del TAM y la tabla 1 resume cada constructo de dicho modelo.

Podemos encontrar estudios que evalúan el TAM como un método eficaz para predecir la aceptación de una tecnología. Sin embargo, Yu, Ha, Choi y Rho (2005) identificaron la falta de un factor con implicaciones sociales en el modelo que influyera en la actitud de los usuarios.

\section{Figura 1. TAM}

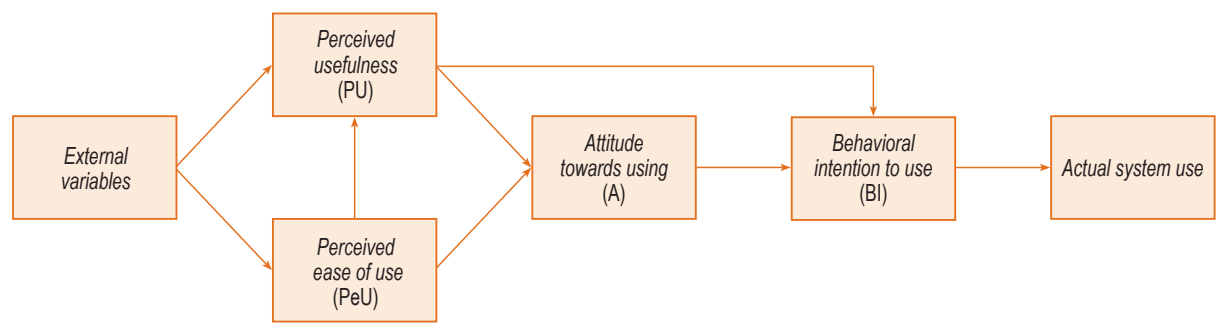

Fuente: conceptos citados por Davis (1985).

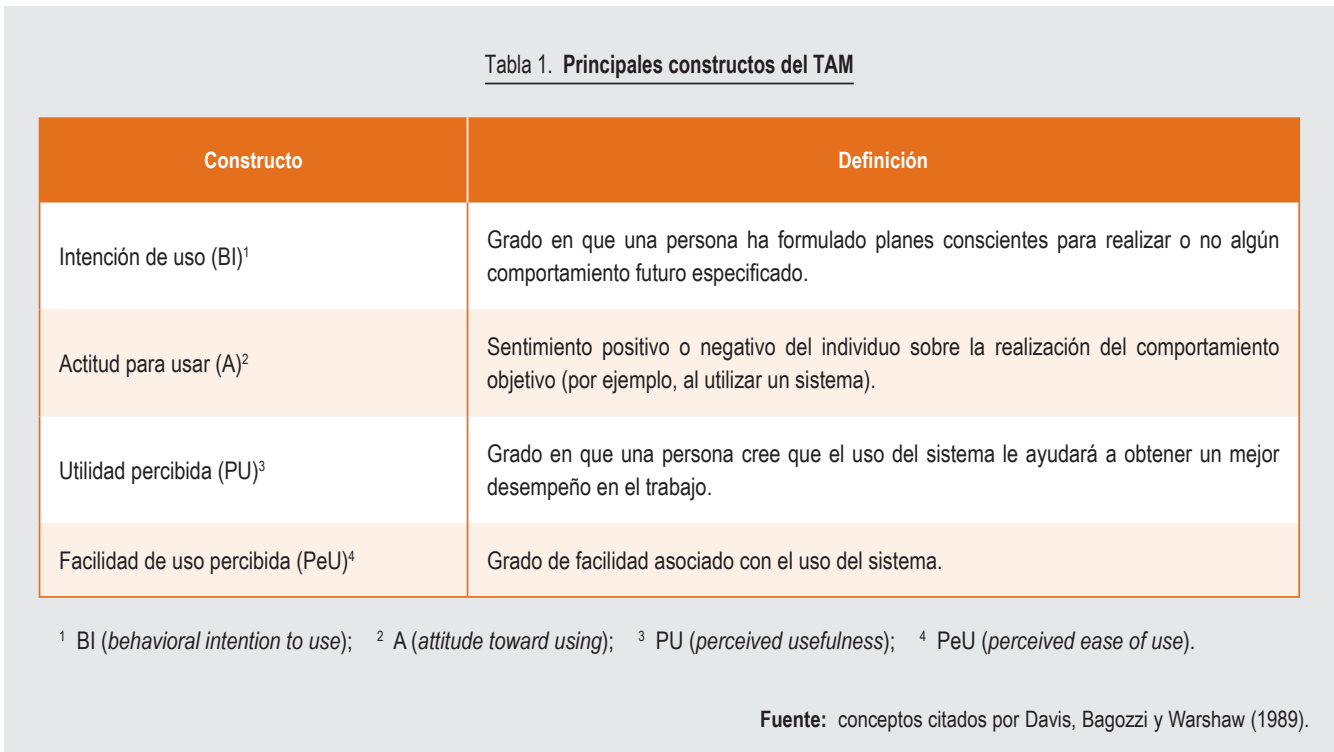

${ }^{4}$ TRA (theory of reasoned action). 
Venkatesh y Davis (2000) añadieron los factores sociales y extendieron el modelo original, llamándolo TAM 2 (véase figura 2). El objetivo principal de la extensión fue incluir algunas variables sociales que tuvieran una implicación directa en los constructos de «utilidad percibida» (PU) e «intención de uso» (IU) $)^{5}$.

EI TAM también se ha implementado en el entorno organizacional para determinar el grado de aceptación de la tecnología. Venkatesh y Bala (2008) implementaron un modelo basado en el TAM para ayudar a la toma de decisiones en las organizaciones. Este modelo se llamó TAM 3, como una extensión de sus predecesores. El modelo combina variables del TAM 2, incorporando variables que influyan en el constructo de «facilidad de uso percibida» (PeU), de tal manera que permita entender cómo podría mejorar la adopción y el uso de la tecnología en las organizaciones.

En este contexto, Chen, Chen y Yen (2011) se enfocan en estudiar el impacto de la variable self-efficacy (entendida como la autocapacidad de emplear un dispositivo sin ayuda) en un estudio a través del uso de dispositivos móviles. En sus resultados observan que la variable estudiada desempeña un papel importante y tiene un impacto positivo en el constructo de «facilidad de uso percibida» (PeU), mientras que afecta parcialmente a la «utilidad percibida» (PU). La figura 3 muestra el diagrama del TAM con sus principales constructos y variables.

Figura 2. TAM 2

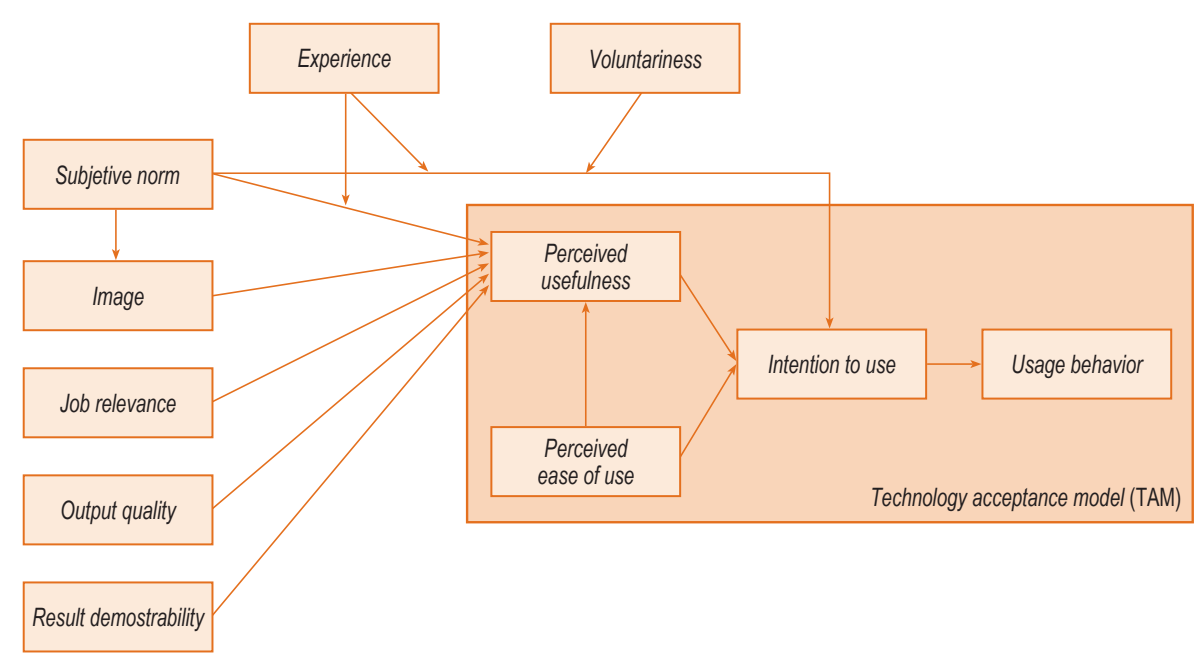

Fuente: Venkatesh y Davis (2000)

\footnotetext{
${ }^{5} \mathrm{IU}$ (intention to use).
} 
Figura 3. TAM 3

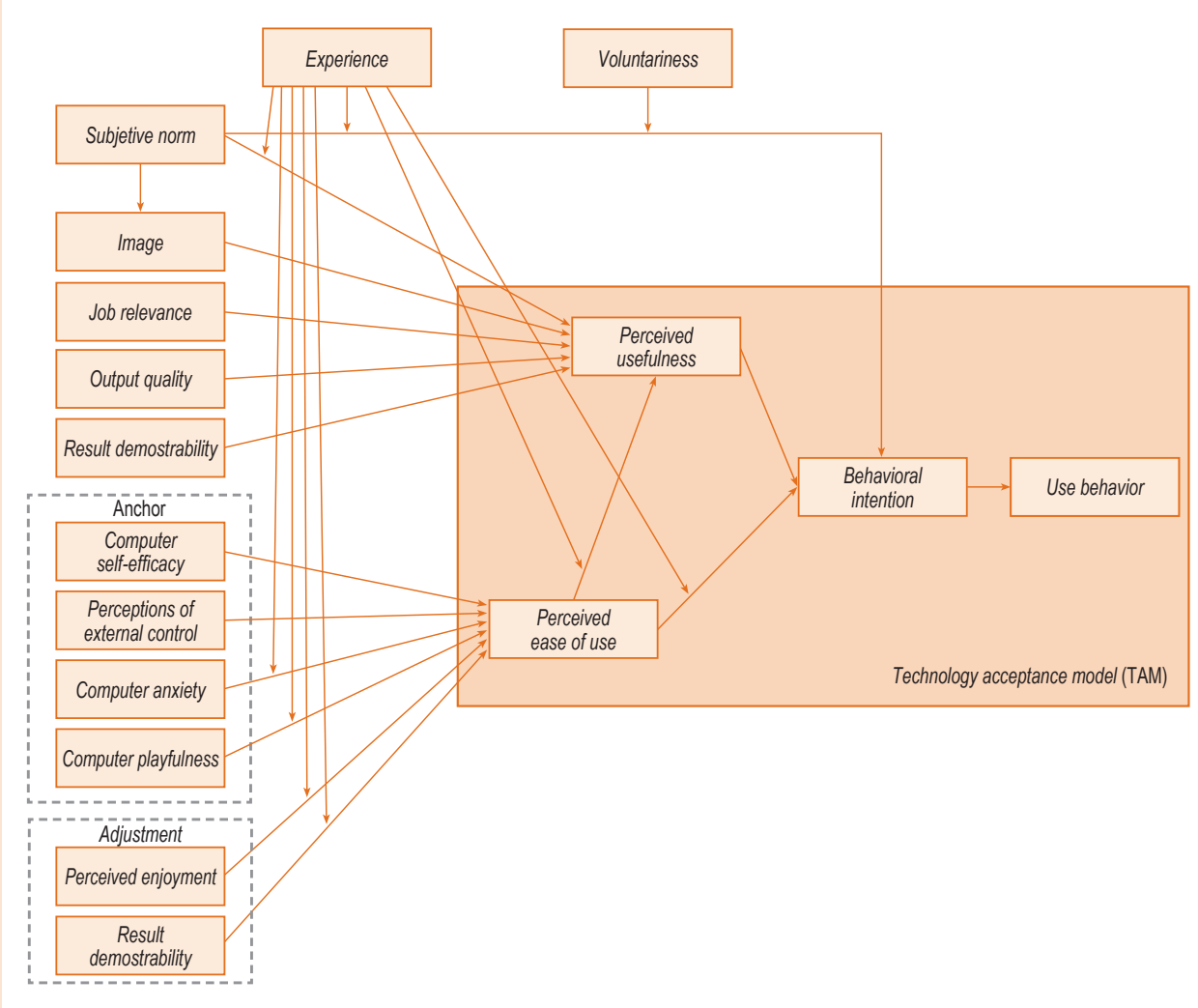

Fuente: transcripción de Venkatesh y Bala (2008, p. 280).

El modelo TAM ha sido implementado en varios estudios relacionados con la evaluación de la aceptación tecnológica. Consideramos el modelo TAM como una herramienta flexible para explicar el comportamiento del usuario final. Creemos también que este método puede proporcionar nuevas visiones generales en el área de innovación, por lo tanto, hemos seleccionado este modelo para apoyar el trabajo de investigación cuantitativo y, además, para comprender los comportamientos de los usuarios finales. Los resultados obtenidos en este modelo podrían contribuir a identificar los factores críticos de éxito de la adopción de una tecnología, teniendo en cuenta que estos factores deben influir ampliamente en cualquier proceso de innovación tecnológica en el ámbito del m-learning.
Podemos encontrar estudios de investigación sobre el uso de sistemas de formación en línea basados en ordenadores personales (Deng y Tavares, 2013); sin embargo, las investigaciones en la aceptación de tecnologías móviles no son tan comunes.

Algunos de los dispositivos móviles que se pueden utilizar para el aprendizaje son, sin lugar a dudas, los ordenadores portátiles, las tablets y los smartphones; sin embargo, los teléfonos inteligentes son la tecnología móvil más utilizada hoy en día a nivel mundial, razón por la que en nuestra investigación hemos elegido este dispositivo. Al igual que con las tecnologías móviles, podemos encontrar muchos sistemas de e-learning diferentes. Centraremos nuestra investigación en sistemas de aprendizaje institucional en línea, tales como Moodle. 


\subsection{Escalas de medición de variables}

Para la elaboración de las escalas, se realizó una exhaustiva revisión bibliográfica, basada en Davis (1985), y se desarrolló el siguiente procedimiento (véase tabla 2):

- Recolección de ítems en cada variable.

- Selección de ítems por los investigadores, considerando el objeto de estudio (m-learning).
- Colaboración con expertos en el área para analizar las escalas y definir los ítems finales.

Las escalas de calificación fueron definidas usando una escala Likert de 7 puntos, con un valor mínimo de 1 (muy en desacuerdo) y un valor máximo de 7 (totalmente de acuerdo).

\section{Tabla 2. Relación de variables estudiadas}

\begin{tabular}{|c|c|}
\hline Constructo & Elemento \\
\hline Intención de uso (BI) & $\begin{array}{l}\text { BI_1. Estoy dispuesto a utilizar el sistema de formación en línea de mi institución desde } \\
\text { mi smartphone. } \\
\text { BI_2. Recomendaria a otros usuarios utilizar el sistema de formación en línea de mi } \\
\text { institución desde sus teléfonos inteligentes. } \\
\text { BI_3. Creo que voy a utilizar frecuentemente el sistema de formación en línea de mi } \\
\text { institución desde mi smartphone. }\end{array}$ \\
\hline Utilidad percibida (PU) & $\begin{array}{l}\text { PU_1. El uso de mi smartphone para trabajar en el sistema de formación en línea de mi } \\
\text { institución mejoraría mi aprendizaje. } \\
\text { PU_2. Usar mi smartphone para trabajar en el sistema de formación en línea de mi } \\
\text { institución mejorará mi productividad y desarrollará mi aprendizaje. } \\
\text { PU_3. Usar mi smartphone para trabajar en el sistema de formación en línea de mi } \\
\text { institución me ayudaría a completar mis actividades de aprendizaje más rápido. } \\
\text { PU_4. Me resulta útil utilizar mi smartphone para acceder al sistema de formación en línea } \\
\text { de mi institución. }\end{array}$ \\
\hline Facilidad de uso percibida (PeU) & $\begin{array}{l}\text { PeU_1. Es sencillo aprender a utilizar el sistema de formación en línea de mi institución } \\
\text { desde mi smartphone. } \\
\text { PeU_2. Es sencillo encontrar información y actividades en el sistema de formación en } \\
\text { línea de mi institución desde mi smartphone. } \\
\text { PeU_3. Es sencillo convertirse en experto utilizando el sistema de formación en línea de } \\
\text { mi institución desde mi smartphone. }\end{array}$ \\
\hline Gozo percibido (PEN) ${ }^{1}$ & $\begin{array}{l}\text { PEN_1. El uso del sistema de formación en línea de mi institución a través de mi } \\
\text { smartphone hará que mis actividades de aprendizaje sean más agradables. } \\
\text { PEN_2. Me encanta usar el sistema de formación en línea de mi institución desde mi } \\
\text { smartphone. } \\
\text { PEN_3. El uso del sistema de formación en línea de mi institución mejorará mi motivación } \\
\text { académica si accedo desde mi smartphone. }\end{array}$ \\
\hline & $\ldots / \ldots$ \\
\hline
\end{tabular}


Tabla 2. Relación de variables estudiadas (cont.)

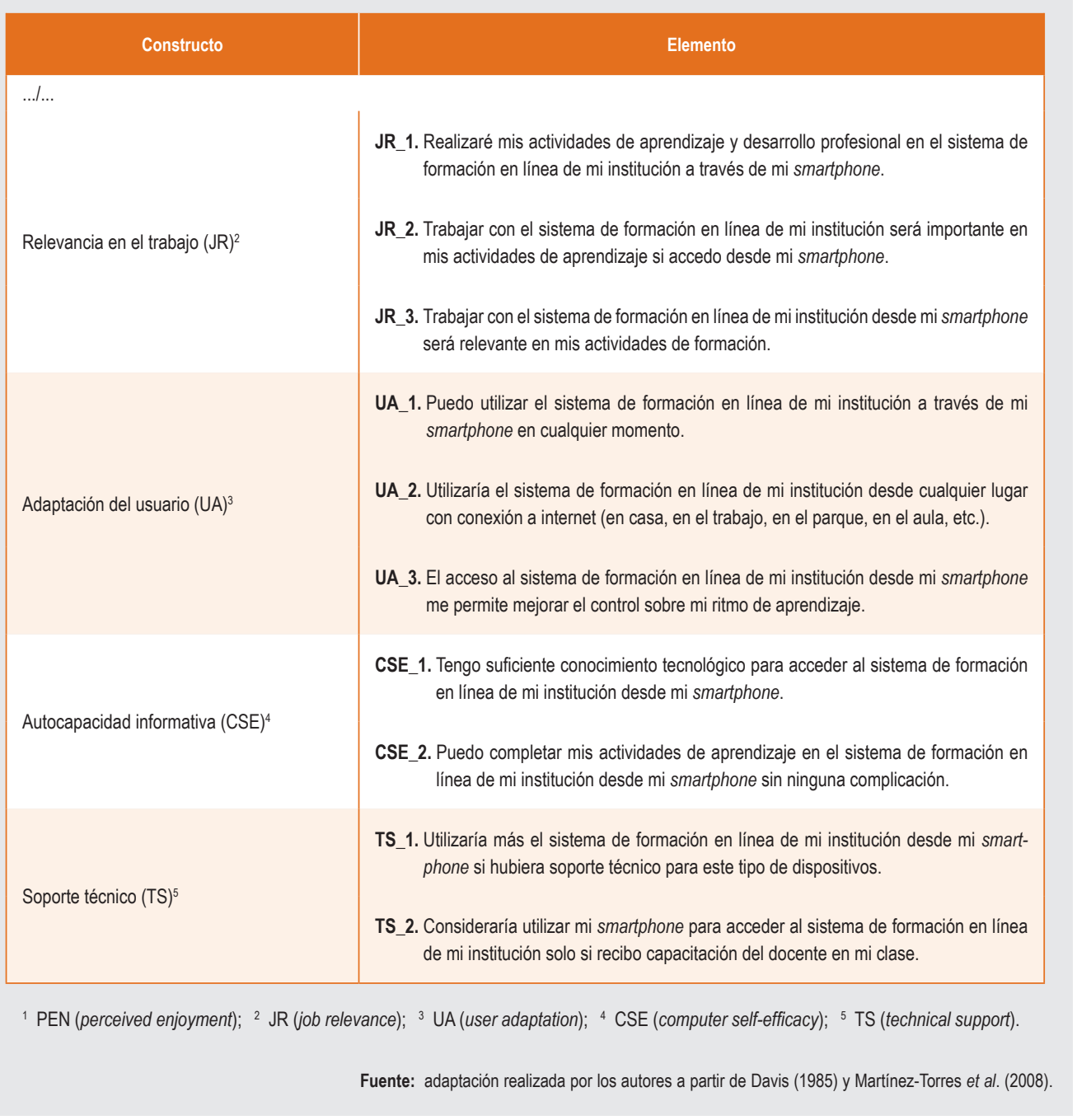

\subsection{Desarrollo de hipótesis de investigación}

Como se pudo identificar en los párrafos anteriores, el núcleo central del TAM radica en los constructos de «utilidad percibida» (PU) y «facilidad de uso percibida» (PeU). Es decir, el modelo sostiene que tanto la percepción de utilidad como de facilidad de uso que experimenten los usuarios de un sistema o una tecnología harán que estos acepten y sean más propensos a utilizar dicho sistema o tecnología.
Con el fin de reconocer la aceptación tecnológica de m-learning y, basado en el núcleo central del TAM, proponemos las siguientes hipótesis en nuestra investigación:

- H1. La «utilidad percibida» (PU) tiene un impacto positivo en los usuarios del sistema de formación en línea institucional a través del smartphone. 
- H1.1. La «utilidad percibida» (PU) tendrá un efecto positivo en la «intención de uso» (BI) al utilizar el sistema de formación en línea institucional a través del smartphone.

- H2. La «facilidad de uso percibida» (PeU) tiene un impacto positivo en los usuarios del sistema de formación en línea institucional a través del smartphone.

- H2.1. La «facilidad de uso percibida» (PeU) tendrá un efecto positivo en la «intención de uso» (BI) al utilizar el sistema de formación en línea institucional a través del smartphone.

- H2.2. La «facilidad de uso percibida» (PeU) tendrá un efecto positivo en la «utilidad percibida» (PU) al usar el sistema de formación en línea institucional a través del smartphone.

Como se explicó anteriormente, cada una de las variables del TAM asociadas a la evaluación del modelo influyen en la percepción individual sobre la facilidad de uso del sistema o la tecnología. En esta investigación, evaluaremos la percepción de los usuarios sobre el uso del smartphone como herramienta de aprendizaje. Venkatesh y Davis (2000, p. 191) definen la variable «relevancia en el trabajo» (JR) como «el grado en que una persona percibe que el sistema objetivo es relevante para su trabajo». Kim (2008) concluye que la mayoría de las personas están dispuestas a adoptar una tecnología útil, pero esta aceptación no está ligada a la habilidad de utilizarla y a la experiencia previa de las TIC. Por lo tanto, la aceptación de dispositivos móviles para el uso de sistemas de aprendizaje en línea requiere una plataforma tecnológica que permita a los usuarios conceder acceso desde cualquier lugar y en cualquier momento. Sobre esta base, desarrollamos la siguiente hipótesis.

- H3. El uso de un smartphone tendrá una relación positiva en la «utilidad percibida» (PU) del usuario para acceder y trabajar en el sistema de formación en línea institucional.

- H3.1. La variable «relevancia en el trabajo» (JR) tendrá un efecto positivo en la «utilidad percibida» (PU).
Se han identificado otras variables que apoyan al constructo "facilidad de uso percibida» (PeU) y que permiten lograr una mejor explicación sobre la aceptación de la tecnología. Chow, Herold, Choo y Chan (2012) utilizaron la variable «autocapacidad informática» (CSE). Estos investigadores demuestran que esta variable tiene un impacto positivo sobre la «facilidad de uso percibida» (PeU) y afecta también parcialmente a la «utilidad percibida» (PU). La variable "autocapacidad informática» (CSE) se define como "el grado en que una persona cree que él o ella tiene la capacidad de realizar una tarea o trabajo específico usando un ordenador» (Venkatesh y Bala, 2008). De acuerdo con esto, proponemos la siguiente hipótesis.

- H4. Los usuarios del sistema de formación en línea institucional perciben que tienen las habilidades necesarias para trabajar en este entorno a través de su smartphone.

- H4.1. La «autocapacidad informática» (CSE) tendrá un efecto positivo en la «utilidad percibida» (PU) del usuario cuando realice sus actividades online a través de su smartphone.

- H4.2. La «autocapacidad informática» (CSE) tendrá un efecto positivo en la «facilidad de uso percibida» (PeU) del usuario cuando realice sus actividades online a través de su smartphone.

Holden y Rada (2011, p. 345) señalan que «el TAM original fue creado antes de que la demanda de uso de la tecnología aumentara, por lo que no incluye medidas esenciales relacionadas con la intención de uso de la tecnología de los usuarios». En nuestra investigación se incorporó la variable «adaptación del usuario» (UA), que podría definirse como «el grado de adaptación de una herramienta o sistema en las actividades de los usuarios utilizando diferentes medios o dispositivos». Con la incorporación de esta variable en nuestro modelo, pretendemos demostrar que la tecnología móvil podría adaptarse a las necesidades de los usuarios en sus actividades de sistemas de formación en línea, por lo que supondremos que existe un efecto positivo hacia los constructos «utilidad percibida» (PU) y "facilidad de uso percibida» $(\mathrm{PeU})$. En relación con ello, proponemos como hipótesis las siguientes declaraciones. 
- H5. Los usuarios consideran que el uso de smartphones para trabajar con el sistema de formación en línea institucional tendrá una buena adaptación en sus actividades.

- H5.1. La «adaptación del usuario» (UA) a las actividades en el sistema de formación en línea institucional a través del smartphone tendrá un efecto positivo en la «utilidad percibida» (PU) de los usuarios.

- H5.2. La «adaptación del usuario» (UA) a las actividades en el sistema de formación en línea institucional a través de un smartphone tendrá un efecto positivo en la "facilidad de uso percibida» (PeU) de los usuarios.

Las organizaciones podrían incluir en sus estrategias de m-learning algún tipo de servicio de apoyo para sus usuarios con el fin de resolver problemas técnicos. Incluimos en nuestro modelo una variable denominada «soporte técnico» (TS). Ngai, Poon y Chan (2007, p. 262), citados en Cheung y Vogel, (2013, p. 164), señalan que «la asistencia técnica tiene un efecto directo y significativo sobre los constructos «utilidad percibida» (PU) y «facilidad de uso percibida» (PeU)». Venkatesh, Morris, Davis y Davis (2003, p. 453) definen la variable «condiciones facilitadoras» $(F C)^{6}$ como «el grado en que un individuo percibe que una estructura organizativa y técnica brinda el soporte hacia el uso del sistema». Terzis y Economides (2011) subrayan que esta variable puede denominarse «soporte técnico», «factores de recursos», "política y normativa», etc. Para este trabajo de investigación consideraremos utilizar la denominación propuesta por Terzis y Economides para la variable "condiciones facilitadoras» (FC), estableciéndola como «soporte técnico» (TS). De esta manera, cualquier organización debe tener un procedimiento para ayudar a los usuarios con los problemas que se deriven del uso de sistemas y tecnologías. Bajo estos supuestos, formulamos la siguiente hipótesis.

- H6. Los usuarios del sistema de formación en línea institucional que reciben «soporte técnico» (TS) para los problemas de la app tendrán una percepción positiva sobre la utilidad percibida (PU) y la facilidad de uso percibida (PeU).

\footnotetext{
${ }^{6} \mathrm{FC}$ (facilitating condictions).
}

- H6.1. La variable «soporte técnico» (TS) tendrá una relación positiva en la utilidad percibida (PU) del usuario del sistema de formación en línea institucional a través del smartphone.

- H6.2. La variable «soporte técnico» (TS) tendrá una relación positiva en la facilidad de uso percibida (PeU) del usuario del sistema de formación en línea institucional a través del smartphone.

Es lógico pensar que los dispositivos móviles sean considerados como herramientas individuales o personales. Estas herramientas permiten a los usuarios tener experiencias individuales (usuario-dispositivo); por ejemplo, leer un documento, hacer fotografías o navegar por internet buscando información específica. Además de ello, estos dispositivos también permiten realizar actividades de colaboración mediante conexión a internet y servidores web. Para este estudio decidimos evaluar si existe una relación entre el usuario y el sistema de formación en línea institucional; es decir, creemos que al usuario debe parecerle agradable y ha de disfrutar del tiempo que pasa en el sistema. De esta manera, Davis, Bagozzi y Warshaw (1992, p. 1.113) definen la variable del «gozo percibido» (PEN) como «el grado en el que usar un sistema específico es percibido como agradable en sí mismo». Esta variable se ha utilizado comúnmente para describir la «utilidad percibida» (PU) y la "facilidad de uso percibida» (PeU). MartínezTorres et al. (2008) señalan la existencia de algunas variables, tales como «gozo», «herramientas del usuario», «difusión», «metodología» y «adaptación del usuario», sugiriendo que estas pueden tener implicaciones en la motivación de los estudiantes hacia el uso de una herramienta de aprendizaje. Sobre la base de todos estos hallazgos, proponemos la siguiente hipótesis.

- H7. Los usuarios disfrutan trabajando en el sistema de formación en línea institucional a través de su smartphone.

- H7.1. La variable «gozo percibido» (PEN) tendrá una relación positiva con la «intención de uso» (BI) de los usuarios en el sistema de formación en línea institucional si utilizan su smartphone para acceder a este. La figura 4 muestra el modelo de investigación, sus hipótesis y construcciones. 


\section{Figura 4. Modelo de aceptación tecnológica propuesto para esta investigación}

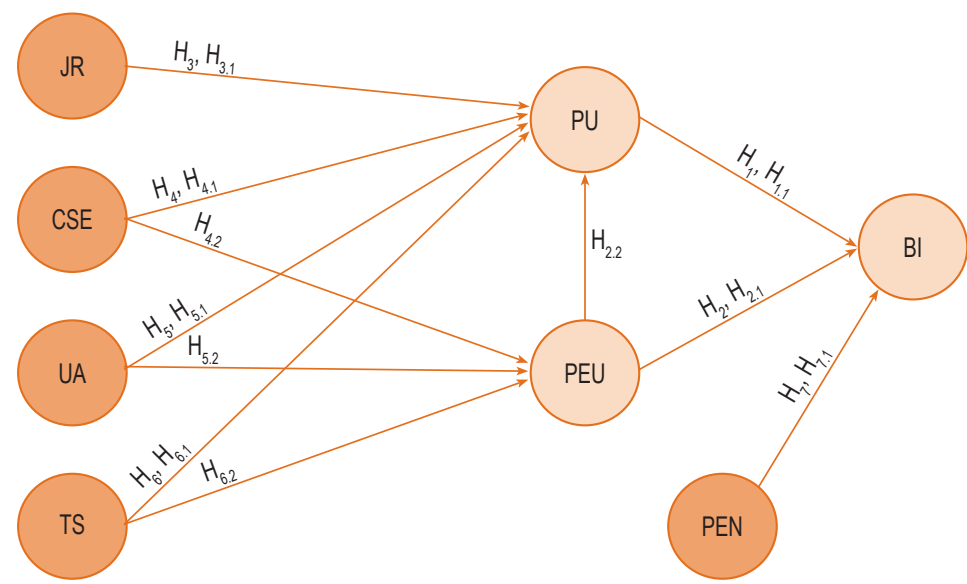

Fuente: elaboración propia a partir de la literatura existente en el área.

\section{METODOLOGÍA DE INVESTIGACIÓN}

Los modelos de ecuaciones estructurales son un método de investigación aceptado en esta área. Por ejemplo, Caballero (2006) señala dos métodos estadísticos para explicar la relación entre procesos y departamentos de organización:

- Modelo basado en covarianza (CB-SEM)

- Método por mínimos cuadrados parciales (PLS-SEM).

Hair, Sarstedt, Ringle y Mena (2011, p. 415) discuten las principales diferencias entre PLS-SEM y CB-SEM y señalan que el primer modelo «maximiza la varianza explicada de las variables latentes endógenas mediante la estimación de relaciones de modelos parciales en una secuencia iterativa de mínimos cuadrados ordinarios $\mathrm{OLS}^{9}$ », mientras que el segundo «estima los parámetros del modelo para minimizar la discrepancia

\footnotetext{
${ }^{7}$ CB-SEM (covariance-based structural equation modeling).

${ }^{8}$ PLS-SEM (partial least squares-structural equation modeling).

${ }^{9}$ OLS (ordinary least squares).
}

entre las matrices de covarianza estimadas y de muestra». Para este trabajo de investigación hemos considerado utilizar el método por PLS, ya que se caracteriza por su flexibilidad para modelar diferentes tipos de problemas estadísticos basados en regresión lineal con un alto grado de dimensionalidad y multicolinealidad y pocas observaciones. Este método ha sido ampliamente utilizado en investigaciones que requieren de análisis multivariantes, en especial, aquellas con enfoque de ciencias sociales, tales como negocios y marketing (Henseler et al., 2014). En este sentido, Hair, Sarstedt, Ringle y Mena (2011) señalan algunas ventajas y desventajas del PLS. Las más destacadas son:

- Es adecuado para aplicaciones en las que las hipótesis no pueden realizarse plenamente y que se refiere frecuentemente como de libre distribución softmodeling approach (estadística no paramétrica).

- Se puede utilizar con algunas restricciones en medidas reflexivas y formativas, mientras que el CBSEM solo es aplicable a la especificación externa formativa bajo ciertos criterios. 
- Puede utilizarse en modelos complejos sin necesidad de centrarse en problemas de estimación, mientras que el CB-SEM restringe comúnmente este tipo de situaciones.

Algunas desventajas del PLS son las siguientes:

- Se centra en maximizar y optimizar modelos en dos etapas: en primer lugar, el modelo de medición, y después, los coeficientes de rutas se calculan en el modelo estructural. Esto hace que primero se deban evaluar las características de los modelos y determinar cuál de estas son inaceptables.

- No se asocia ninguna suposición distributiva, por lo que no puede ser retransmitida en el marco deductivo clásico, debiendo considerarse la predicción no paramétrica de los criterios de evaluación, así como los procedimientos de remuestreo para evaluar la adecuación de las estructuras de los modelos parciales.

\section{DISEÑO DE LA INVESTIGACIÓN}

Resulta complicado determinar cuáles son los factores críticos del éxito de una innovación educativa desde el punto de vista de la aceptación tecnológica: educación elemental, educación permanente, formación profesional, educación superior, aprendizaje permanente, etc., por lo que realizar un estudio que abarque en su totalidad el espectro educativo sería bastante complicado. Debido a esta complejidad, centramos nuestra investigación en una IES local. Consideramos que las IES son innovadoras y representan un factor clave para el desarrollo de la sociedad del conocimiento.

En el entorno de la educación superior, seleccionamos una muestra de estudiantes de la Universitat Rovira i
Virgili durante los meses de abril y mayo de 2014 con la finalidad de obtener datos que permitieran medir nuestro caso de estudio. Adaptamos las escalas de medida revisadas en la literatura sobre el sistema de formación en línea institucional, que, para este caso, fue el LMS Moodle. Existen muchos dispositivos móviles en el mercado (netbooks, e-readers, tabletas, smartphones, etc.), pero en esta investigación nos hemos centrado en los smartphones, ya que es uno de los dispositivos móviles con mayor penetración. El sistema de formación institucional está preparado para acceder a él a través de tecnologías móviles, utilizando las apps o el navegador. La figura 5 muestra la aplicación de Moodle para Android e iOS.

Figura 5. Interfaz de aplicación Moodle para Android y Apple iOS
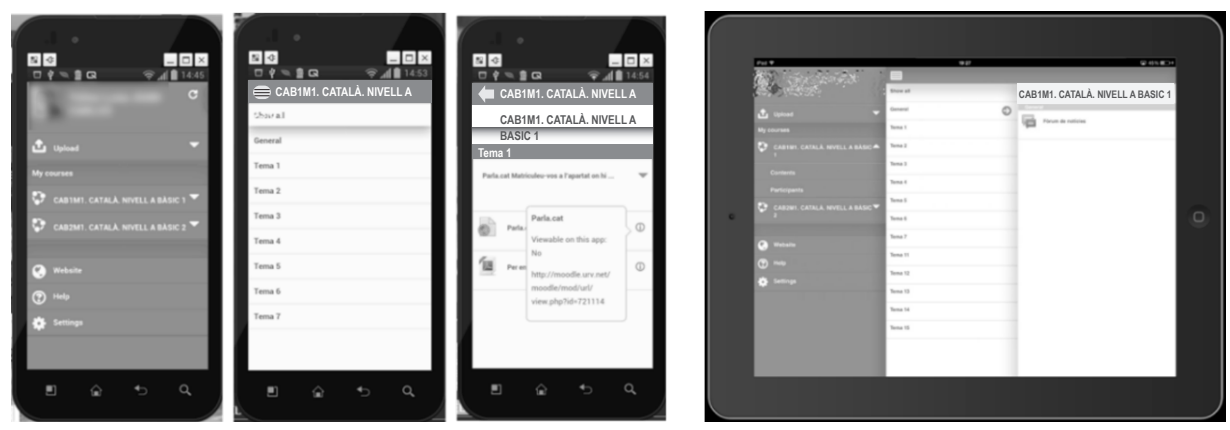

Fuente: elaboración propia. 
Durante el periodo anteriormente mencionado, Moodle registró 4.598.247 sesiones, con un promedio de páginas por sesión de $9,35 \%$ y un tiempo medio de conexión por sesión en torno a los 9 minutos. Android es el sistema más utilizado, con el $58,83 \%$ del total de sesiones, seguido de Apple iOS con el $39,98 \%$, tal y como podemos ver en la figura 6 .

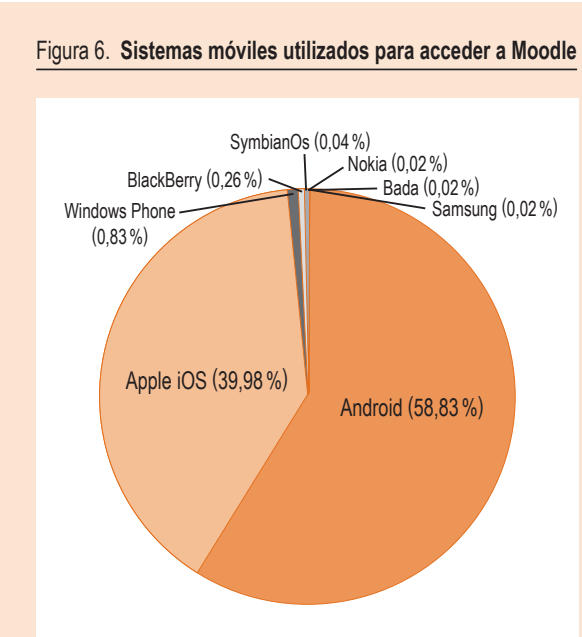

Fuente: elaboración propia a partir de los datos obtenidos de la Universitat Rovira i Virgili.

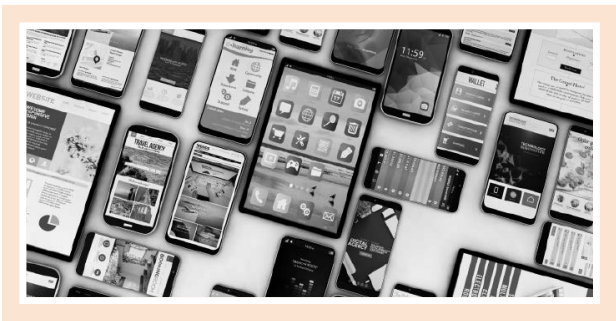

Con el fin de obtener y recopilar los datos, se desarrolló un cuestionario que se aplicó en una sola etapa al final del año académico a través de la línea con el servicio de encuestas de Google Drive. Fue enviado a todos los estudiantes por el servicio de correo electrónico institucional, obteniendo 555 encuestas. Del total de encuestas, 35 de ellas fueron descartadas por su inconsistencia en las respuestas, por lo que, finalmente, se obtuvieron 520 encuestas para el análisis. Los resultados de la muestra de evaluación se pueden observar en la tabla 3.

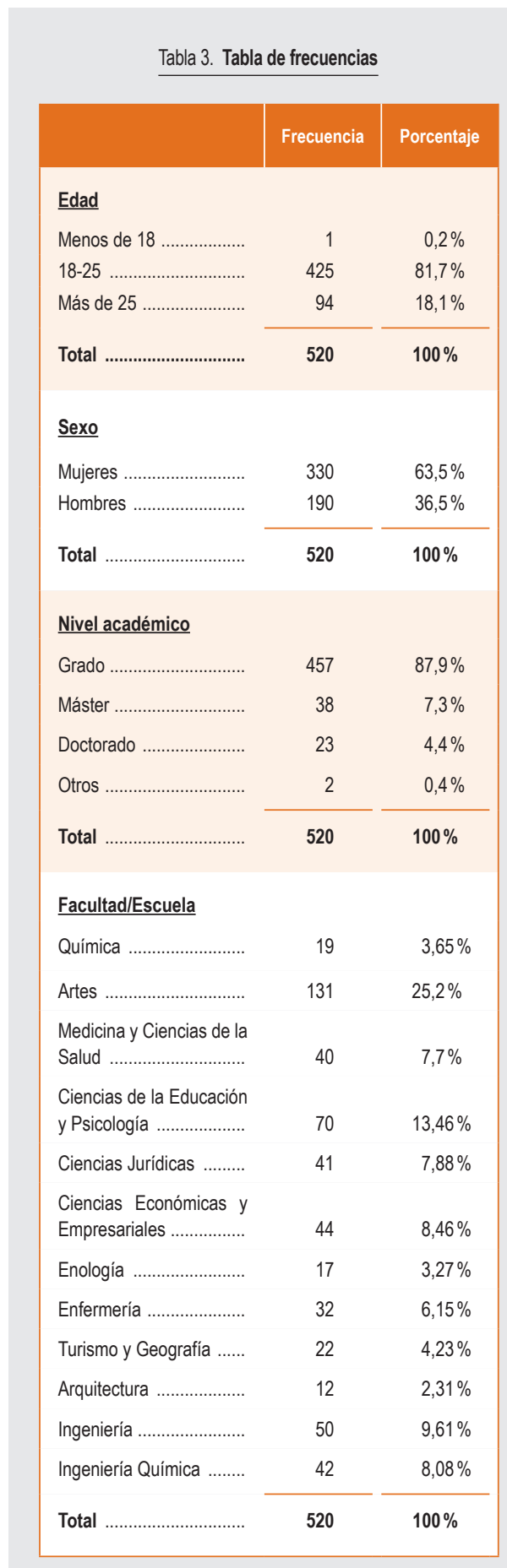

Fuente: elaboración propia 


\section{EVALUACIÓN DEL MODELO PROPUESTO}

Para esta investigación, las relaciones del modelo entre los constructos y sus elementos serán consideradas como reflexivas. De acuerdo con Roldán y Sánchez-Franco (2012), los modelos con medidas reflexivas deben ser evaluados a través de la validez convergente, discriminante, y con la confiabilidad compuesta de los indicadores.

Martínez-Torres et al. $(2008$, p. 7) señalan que «la prueba de validez convergente indica el grado en el que los ítems de una escala, que, en un supuesto dado, se relacionan entre sí, deberían mostrar una correlación fuerte». Las escalas utilizadas para medir la validez convergente del modelo propuesto se basaron en las especificaciones aceptadas en la investigación académica para estos modelos. Todas las cargas factoriales de los indicadores deben ser significativas y superar 0,5 . También se especifica que la fiabilidad compuesta debe ser superior a 0,7 , ya que este valor se considera «modesto» y los valores sugeridos deben ser superiores a 0,8. La varianza media extraída (AVE) ${ }^{10}$ debe ser superior a 0,5 para ser aceptable. La tabla 4 muestra que los valores obtenidos para el modelo de investigación se apoyan en criterios previamente definidos, por lo que se consideran aceptables.

\subsection{Fiabilidad individual}

Para validar el modelo de investigación es necesario aplicar instrumentos que permitan la evaluación de la confiabilidad de cada constructo y que estén basados en la muestra de investigación. Para medir la fiabilidad de los constructos se utiliza el coeficiente alfa de Cronbach. El coeficiente alfa de Cronbach es un elemento de correlación en una medición de escala, es decir, cada elemento debe estar correlacionado entre sí. No existen valores específicos para interpretar este coeficiente, sin embargo, se sugiere que los valores deben ser interpretados de acuerdo con el objetivo propuesto de la investigación. Los intervalos de valores alfa de Cronbach oscilan entre 0 y 1 , es decir, la fiabilidad de la construcción aumentará si el coeficiente se aproxima a 1.

El valor mínimo aceptable para un alfa de Cronbach es de 0,70 y los valores más bajos se considerarán insuficientes. El valor esperado para el alfa de Cronbach es 0,90 y los que superan los valores esperados serán considerados redundantes. En nuestro modelo de investigación se encontró que el valor alfa de Cronbach, en el constructo «autocapacidad informática» (CSE), tenía un valor menor que el criterio esperado; sin embargo, optamos por continuar la evaluación de esta construcción porque observamos que la «varianza media extraída» (AVE) y la fiabilidad compuesta mostraron valores aceptables. La fiabilidad individual se resume en la tabla 4 y las cargas del modelo externo en la tabla 5.

Tabla 4. Análisis de fiabilidad individual

\begin{tabular}{|c|c|c|c|c|}
\hline & $\begin{array}{l}\text { Varianza media } \\
\text { extraída (AVE) }\end{array}$ & Fiabilidad compuesta & $\mathrm{R}^{2}$ & Alfa de Cronbach \\
\hline 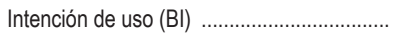 & 0,8461 & 0,9428 & 0,5123 & 0,9091 \\
\hline Autocapacidad informática (CSE) .................. & 0,7066 & 0,8279 & 0 & 0,5863 \\
\hline Relevancia en el trabajo (JR) ........................ & 0,8678 & 0,9516 & 0 & 0,9237 \\
\hline 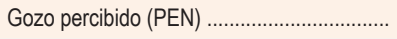 & 0,7999 & 0,923 & 0 & 0,8764 \\
\hline Utilidad percibida (PU) ................................... & 0,7581 & 0,9258 & 0,6584 & 0,892 \\
\hline Facilidad de uso percibida (PeU) ................. & 0,8403 & 0,9404 & 0,3675 & 0,9051 \\
\hline 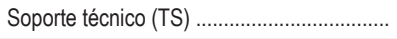 & 0,8014 & 0,8897 & 0 & 0,7549 \\
\hline 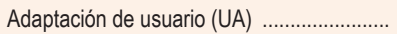 & 0,6879 & 0,8681 & 0 & 0,7707 \\
\hline
\end{tabular}

Fuente: elaboración propia.

10 AVE (average variance extracte). 


\begin{tabular}{|c|c|c|}
\hline \multicolumn{3}{|c|}{ Tabla 5. Relación de cargas factoriales } \\
\hline Constructo & Variable & Cargas \\
\hline \multirow{3}{*}{ Intención de uso (BI) } & BI_1 & 0,9221 \\
\hline & BI_2 & 0,9178 \\
\hline & BI_3 & 0,9196 \\
\hline \multirow{2}{*}{$\begin{array}{l}\text { Autocapacidad informática } \\
\text { (CSE) }\end{array}$} & CSE_1 & 0,8164 \\
\hline & CSE_2 & 0,8640 \\
\hline \multirow{3}{*}{$\begin{array}{l}\text { Relevancia en el trabajo } \\
\text { (JR) }\end{array}$} & JR_1 & 0,9113 \\
\hline & JR_2 & 0,9514 \\
\hline & JR_3 & 0,9314 \\
\hline \multirow{3}{*}{ Gozo percibido (PEN) } & PEN_1 & 0,8941 \\
\hline & PEN_2 & 0,9022 \\
\hline & PEN_3 & 0,8867 \\
\hline \multirow{4}{*}{ Utilidad percibida (PU) } & PU_1 & 0,9101 \\
\hline & PU_2 & 0,9252 \\
\hline & PU_3 & 0,8535 \\
\hline & PU_4 & 0,7872 \\
\hline \multirow{3}{*}{$\begin{array}{l}\text { Facilidad de uso percibida } \\
\text { (PeU) }\end{array}$} & PeU_1 & 0,9098 \\
\hline & PeU_2 & 0,9270 \\
\hline & PeU_3 & 0,9132 \\
\hline \multirow{2}{*}{ Soporte técnico (TS) } & TS_1 & 0,9191 \\
\hline & TS_2 & 0,8707 \\
\hline \multirow{3}{*}{$\begin{array}{l}\text { Adaptación del usuario } \\
\text { (UA) }\end{array}$} & UA_1 & 0,8773 \\
\hline & UA_2 & 0,8570 \\
\hline & UA_3 & 0,7480 \\
\hline \multicolumn{3}{|c|}{ Todas las cargas tienen un valor superior a 0,7 . } \\
\hline
\end{tabular}

\subsection{Validez discriminante}

Martínez-Torres et al. (2008, p. 500) definen la validez discriminante como «el grado en que la variable medida no es un reflejo de alguna otra variable; es decir, es validar que aquellos constructos que no deberán tener relaciones entre sí en realidad no las tengan».

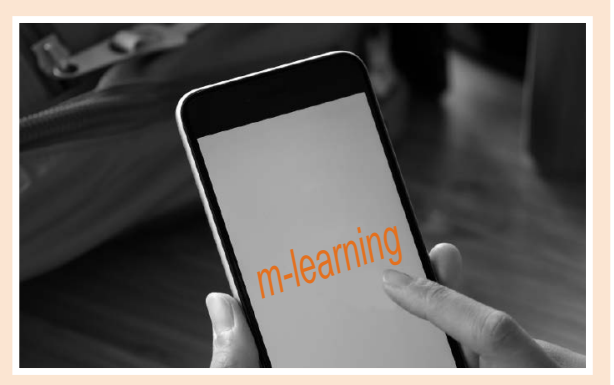

De esta forma, según los criterios de evaluación definidos anteriormente, la validez discriminante se considera como el valor de la raíz cuadrada de la «varianza media extraída» (AVE) en cada constructo. Este valor debe ser mayor en comparación con la correlación de los otros constructos. La tabla 6 muestra los valores de la raíz cuadrada de la "varianza media extraída» (AVE) para el modelo propuesto. Los valores diagonales se soportan con los criterios mencionados anteriormente. Por lo tanto, suponemos que existe una validez discriminante apropiada en cada constructo.

\subsection{Evaluación del modelo estructural}

El modelo estructural y los valores de las rutas se visualizan en la figura 7. Los valores estadísticos significativos se determinaron a través del método de remuestreo (bootstrapping) 11 mediante el cálculo de los valores t-Student. Roldán y Sánchez-Franco (2012) describen el proceso de bootstrapping como el método para generar muestras aleatorias basadas en la muestra original. Este proceso obtendrá los valores de error estándar y t-Student, que pondrán a prueba nuestras hipótesis de investigación.

Roldán y Sánchez-Franco (2012) recomiendan usar un mínimo de 500 muestras y el número de casos (bootstrap) debe ser igual al número de observaciones en el estudio original. Sin embargo, otros autores han aumentado el número de muestreos; por ejemplo, Hair, Ringle y Sarstedt (2011) sugieren el uso de 5.000 remuestreos para tener un mejor apoyo en el contraste de las hipótesis.

\footnotetext{
${ }^{11}$ Según Roldán y Sánchez-Franco (2012, p. 221), el bootstrapping es «una técnica no paramétrica de remuestreo comúnmente utilizada en PLS, que proporciona errores estándar y el estadistico t de los parámetros».
} 


\begin{tabular}{|c|c|c|c|c|c|c|c|c|}
\hline \multicolumn{9}{|c|}{ Tabla 6. Validez discriminante } \\
\hline & $\begin{array}{l}\text { Intención de } \\
\text { uso } \\
\text { (BI) }\end{array}$ & $\begin{array}{c}\text { Autocapacidad } \\
\text { informática } \\
\text { (CSE) }\end{array}$ & $\begin{array}{c}\text { Relevancia en } \\
\text { el trabajo } \\
\text { (JR) }\end{array}$ & $\begin{array}{l}\text { Gozo } \\
\text { percibido } \\
\text { (PEN) }\end{array}$ & $\begin{array}{l}\text { Utilidad } \\
\text { percibida } \\
\text { (PU) }\end{array}$ & $\begin{array}{c}\text { Facilidad de } \\
\text { uso percibida } \\
\text { (PeU) }\end{array}$ & $\begin{array}{l}\text { Soporte } \\
\text { técnico } \\
\text { (TS) }\end{array}$ & $\begin{array}{l}\text { Adaptación } \\
\text { del usuario } \\
\text { (UA) }\end{array}$ \\
\hline Intención de uso (BI) .................... & 0,91984 & & & & & & & \\
\hline Autocapacidad informática (CSE) & 0,37630 & 0,84060 & & & & & & \\
\hline Relevancia en el trabajo (JR) ..... & 0,52690 & 0,37490 & 0,93156 & & & & & \\
\hline Gozo percibido (PEN) .................. & 0,64700 & 0,38150 & 0,81870 & 0,89437 & & & & \\
\hline Utilidad percibida (PU) ................. & 0,66400 & 0,37580 & 0,77640 & 0,80500 & 0,87069 & & & \\
\hline Facilidad de uso percibida (PeU) & 0,52790 & 0,49770 & 0,39140 & 0,47560 & 0,45650 & 0,91668 & & \\
\hline 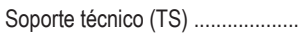 & 0,45440 & 0,20340 & 0,59250 & 0,60240 & 0,57460 & 0,26120 & 0,89521 & \\
\hline Adaptación del usuario (UA) ........ & 0,55690 & 0,47210 & 0,60250 & 0,62120 & 0,61230 & 0,54010 & 0,48830 & 0,82940 \\
\hline
\end{tabular}

Fuente: elaboración propia.

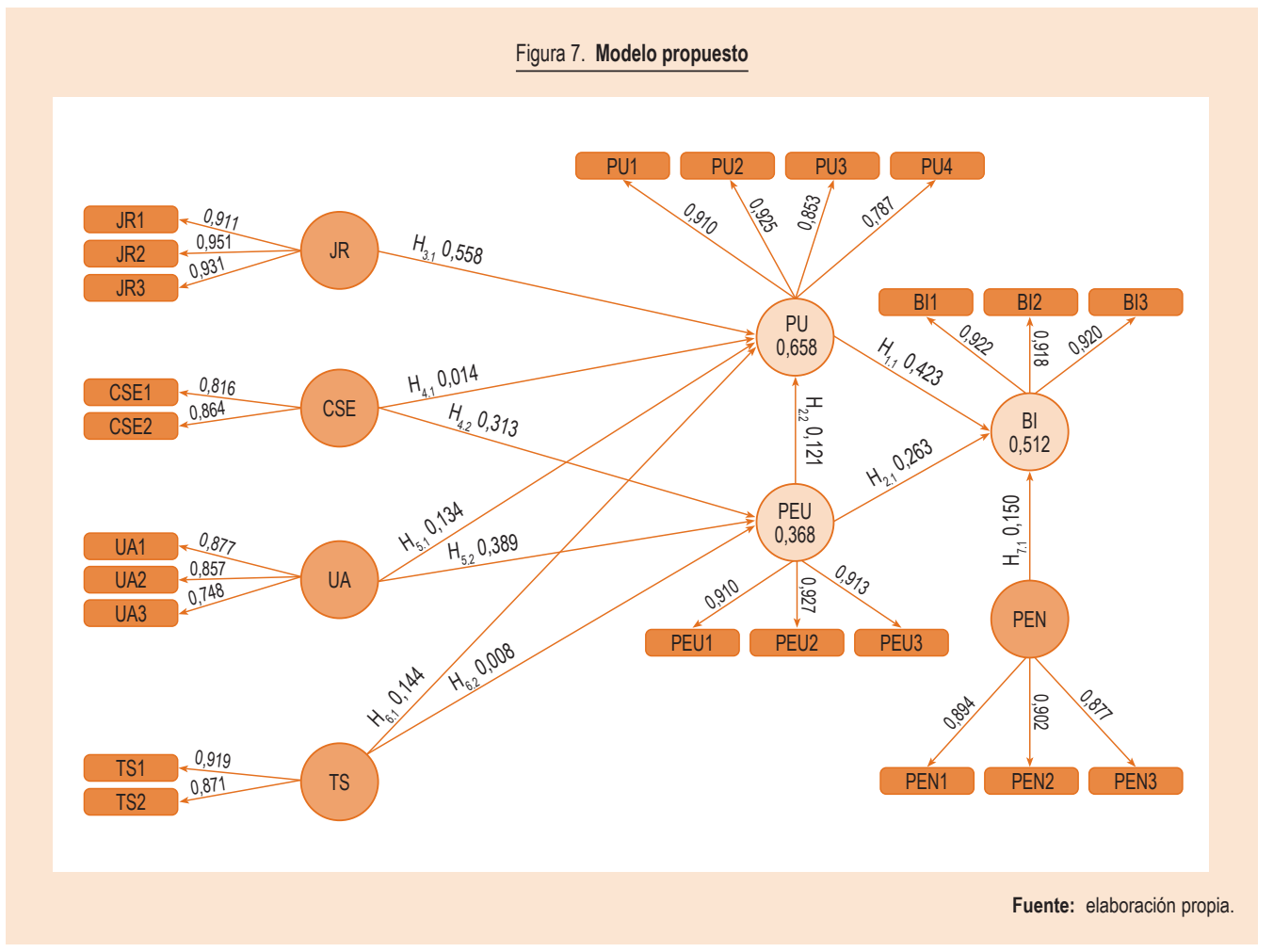


Para este modelo de investigación se considera realizar un bootstrap de 5.000 iteraciones para los 520 casos del estudio. A su vez, los valores obtenidos de la prueba t-Student se utilizarán para contrastar los intervalos de confianza. De acuerdo con la metodología utilizada en Roldán y Sánchez-Franco (2012) siguiendo este criterio: $p<0,05 ; p<0,01$ y $p<0,001$; por lo tanto, el modelo de investigación basado en $\mathrm{t}(\mathrm{N}-1) \rightarrow \mathrm{t}$ $(5.000-1)$; la estadística para una cola prueba sería $\mathrm{t}(0,05 ; 4.999)=1,6451, \mathrm{t}(0,01 ; 4.999)=2,3270 \mathrm{y} \mathrm{t}$ $(0,001 ; 4.999)=3,0902$.

La tabla 7 muestra los resultados del modelo estructural para cada una de las hipótesis. Se puede observar que la mayoría de las hipótesis (H1.1, H2.1, $\mathrm{H} 3.1, \mathrm{H} 4.2, \mathrm{H} 5.1, \mathrm{H} 5.2$ y H6.1) se validan para una $\mathrm{p}$ $<0,001$. Solo dos de las hipótesis del estudio $(H 2.2$ y $\mathrm{H7}$.1) fueron validadas en $p<0,01$. Finalmente, las hipótesis restantes no alcanzaron el valor mínimo de distribución (H4.1 y H6.2), por lo que no se validan en este modelo de investigación.

Existen varios métodos para calcular los intervalos de confianza de bootstrap. Roldán y Sánchez-Franco (2012) sugieren el método de percentiles evaluados en un $2,5 \%$ y en un $97,5 \%$ en sus valores respectivos. La tabla 8 muestra que en nuestro modelo de investigación todas las hipótesis se aceptan, con excepción de las hipótesis H4.1 y H6.2, que no se validan bajo esta medición. Esas hipótesis no fueron validadas porque el intervalo de confianza contiene valores negativos para el menor y positivos para el mayor, por lo tanto, hay concordancia en ambos resultados de las técnicas empleadas.

Regularmente, en los estudios en los cuales se deben realizar regresiones lineales, el coeficiente de determinación $\left(R^{2}\right)$ es una herramienta que permite comprender la varianza explicada de los constructos del modelo. Roldán y Sánchez-Franco (2012, p. 205)

\begin{tabular}{|c|c|c|c|c|c|}
\hline & Hipótesis & Efecto & Coeficientes & Valor & Soportado \\
\hline $\mathrm{H} 1$ & $\mathrm{H} 1.1: \mathrm{PU} \rightarrow \mathrm{BI}$ & + & 0,4235 & $6,7959^{* * *}$ & Si \\
\hline $\mathrm{H} 2$ & $\begin{array}{l}\mathrm{H} 2.1: \mathrm{PeU} \rightarrow \mathrm{BI} \\
\mathrm{H} 2.2: \mathrm{PeU} \rightarrow \mathrm{PU}\end{array}$ & + & $\begin{array}{l}0,2631 \\
0,1215\end{array}$ & $\begin{array}{l}6,8607^{* * *} \\
2,501^{\star *}\end{array}$ & $\begin{array}{l}\text { Si } \\
\text { Si }\end{array}$ \\
\hline $\mathrm{H} 3$ & $\mathrm{H} 3.1: \mathrm{JR} \rightarrow \mathrm{PU}$ & + & 0,5575 & $12,3658^{\star * *}$ & sí \\
\hline $\mathrm{H} 4$ & $\begin{array}{l}\mathrm{H} 4.1: \mathrm{CSE} \rightarrow \mathrm{PU} \\
\mathrm{H} 4.2: \mathrm{CSE} \rightarrow \mathrm{PeU}\end{array}$ & + & $\begin{array}{l}0,0137 \\
0,3126\end{array}$ & $\begin{array}{l}0,4034 \\
6,1512^{* \star *}\end{array}$ & $\begin{array}{l}\text { No } \\
\text { Si }\end{array}$ \\
\hline $\mathrm{H} 5$ & $\begin{array}{l}\mathrm{H} 5.1 \mathrm{UA} \rightarrow \mathrm{PU} \\
\mathrm{H} 5.2 \mathrm{UA} \rightarrow \mathrm{PeU}\end{array}$ & + & $\begin{array}{l}0,1338 \\
0,3887\end{array}$ & $\begin{array}{l}3,1478^{* \star \star} \\
6,4734^{\star \star \star}\end{array}$ & $\begin{array}{l}\text { Si } \\
\text { Si }\end{array}$ \\
\hline H6 & $\begin{array}{l}\text { H6.1: TS } \rightarrow \mathrm{PU} \\
\mathrm{H6} 62: \mathrm{TS} \rightarrow \mathrm{PeU}\end{array}$ & + & 0,1444 & $\begin{array}{l}3,8542^{\star * \star} \\
0,1697\end{array}$ & Sí \\
\hline $\mathrm{H} 7$ & H7.1: $\mathrm{PEN} \rightarrow \mathrm{BI}$ & + & 0,1498 & $2,4331^{* *}$ & Si \\
\hline
\end{tabular}

${ }^{*} p<0,05 ;{ }^{* *} p<0,01 ;{ }^{* * *} p<0,001$.

Basado en la prueba $\mathrm{t}(4.999)$ para una cola; por lo tanto, $\mathrm{t}(0,05 ; 4.999)=1,6451 ; \mathrm{t}(0,01 ; 4.999)=2,3270 ; \mathrm{t}(0,001 ; 4.999)=3,0918$.

Fuente: elaboración propia. 
Tabla 8. Prueba por percentiles al $97,2 \%$

\begin{tabular}{|c|c|c|c|c|c|c|}
\hline & Hipótesis & Efecto & Coeficientes & Mínimo $(2,5 \%)$ & Máximo (97,5\%) & Soportado \\
\hline $\mathrm{H} 1$ & $\mathrm{H} 1.1: \mathrm{PU} \rightarrow \mathrm{BI}$ & + & 0,4235 & 0,2959975 & 0,5392125 & Sí \\
\hline \multirow{2}{*}{$\mathrm{H} 2$} & $\mathrm{H} 2.1: \mathrm{PeU} \rightarrow \mathrm{BI}$ & + & 0,2631 & 0,1867 & 0,3392 & Sí \\
\hline & $\mathrm{H} 2.2: \mathrm{PeU} \rightarrow \mathrm{PU}$ & + & 0,1215 & 0,028895 & 0,2213 & Sí \\
\hline H3 & $\mathrm{H} 3.1: \mathrm{JR} \rightarrow \mathrm{PU}$ & + & 0,5575 & 0,4715975 & 0,6461025 & Sí \\
\hline \multirow{2}{*}{$\mathrm{H} 4$} & H4.1: CSE $\rightarrow$ PU & + & 0,0137 & $-0,0547075$ & 0,0782025 & No \\
\hline & $\mathrm{H} 4.2: \mathrm{CSE} \rightarrow \mathrm{PeU}$ & + & 0,3126 & 0,2158925 & 0,4132025 & Sí \\
\hline \multirow{2}{*}{ H5 } & $\mathrm{H} 5.1: \mathrm{UA} \rightarrow \mathrm{PU}$ & + & 0,1338 & 0,0512975 & 0,2185 & Sí \\
\hline & $\mathrm{H} 5.2: \mathrm{UA} \rightarrow \mathrm{PeU}$ & + & 0,3887 & 0,2660875 & 0,5015 & Sí \\
\hline \multirow{2}{*}{$\mathrm{H} 6$} & H6.1: TS $\rightarrow$ PU & + & 0,1444 & 0,073495 & 0,21811 & Sí \\
\hline & H6.2: TS $\rightarrow$ PeU & + & 0,0078 & $-0,0813075$ & 0,1003025 & No \\
\hline H7 & H7.1: PEN $\rightarrow$ BI & + & 0,1498 & 0,0380875 & 0,2767025 & Sí \\
\hline
\end{tabular}

Fuente: elaboración propia.

sugieren que «el valor $\mathrm{R}^{2}$ representa una medida del poder predictivo e indica la cantidad de varianza en el constructo en cuestión, lo que se explica por sus variables exógenas». En este sentido, la investigación se basará en el valor del coeficiente de determinación $\left(R^{2}\right)$, por lo que utilizaremos el método PLS-SEM para maximizar la varianza de las variables dependientes con la finalidad de lograr una explicación adecuada del modelo de investigación propuesto, como se recomienda en Hair, Sarstedt, Ringle y Mena (2011).

No hay un criterio fijo para evaluar el coeficiente de determinación; sin embargo, la literatura académica ha establecido algunos criterios. Por ejemplo, en Roldán y Sánchez-Franco (2012) se cita que se deben consideran los valores de $\mathrm{R}^{2}$ como sigue: 0,67 es un valor sustancial, 0,33 es un valor moderado y 0,19 es un valor débil. Además, Hair, Ringle y Sarstedt (2011) sostienen que la selección de criterios $R^{2}$ dependerá del área donde se llevará a cabo el estudio de investigación. Los autores también recomiendan que en áreas relacionadas con el comportamiento del consumidor los valores de 0,20 se puedan considerar altos. En los estudios de mercado, los valores de $R^{2}$ de $0,75,0,50 \circ 0,25$ para las variables latentes endógenas en el modelo estructural podrian describirse como sustanciales, moderados o débiles, respectivamente. En esta investigación, consideramos los valores para el coeficiente de determinación propuestos para el área de mercado.

Los resultados para el coeficiente de determinación en el constructo «utilidad percibida» (PU) fueron los siguientes: «facilidad de uso percibida» (PeU = 5,55\%), «relevancia en el trabajo» (JR $=43,28 \%)$, «autocapacidad informática» (CSE $=0,51 \%$ ), «adaptación del usuario» (UA = $=8,19 \%$ ) y «soporte técnico» (TS $=8,30 \%$ ). La suma total de los predictores explica que el $65,84 \%$ de la población estudiada percibe que el uso de los smartphones tiene aplicación en el sistema de formación institucional; es decir, encuentran utilidad en el empleo del dispositivo, por lo que, estadísticamente, se puede considerar que estos resultados tienen un impacto moderado para aplicación en marketing según los criterios $\mathrm{R}^{2}$. 
Los predictores para el constructo «facilidad de uso percibida» (PeU) fueron los siguientes: «adaptación del usuario» (UA $=20,99 \%$ ), «autocapacidad informática» (CSE $=15,56 \%)$ y «Soporte técnico» (TS = $=0,20 \%)$. La suma total de los predictores fue de $36,75 \%$ para el constructo «facilidad de uso percibida» (PeU), por lo que se puede asumir que un porcentaje mínimo (estadísticamente hablando) presta atención al entorno de trabajo del sistema de formación institucional al acceder desde su smartphone. Sin embargo, cabe mencionar que, aunque los valores son muy bajos, la variable «adaptación del usuario» (UA) sugiere que los encuestados buscan que las actividades que realizan en el sistema puedan adaptarse a las diferentes tecnologías existentes; específicamente, en este estudio, a la tecnología móvil.

Finalmente, los predictores para el constructo «intención de uso» (BI) fueron los siguientes: «utilidad percibida»
( $\mathrm{PU}=28,12 \%)$, «facilidad de uso percibida» ( $\mathrm{PeU}=$ $=13,88 \%$ ) y «gozo percibido» (PEN $=9,22 \%$ ). La suma total de los predictores explica que el $51,23 \%$ de la población encuestada tiene mayor consideración por la utilidad que percibe del sistema que por la facilidad de uso y por el gozo percibido en el momento de decidir si aceptarlo o no.

Roldán y Sánchez-Franco (2012, p. 221) sugieren aplicar la prueba de Stone-Geisser $\left(Q^{2}\right)$ para evaluar la relevancia predictiva de los constructos endógenos con un modelo de medición reflexivo. Los criterios adoptados en esta investigación para evaluar la relevancia predictiva son los propuestos por Roldán y SánchezFranco: un $Q^{2}$ mayor que 0 implica que el modelo tiene relevancia predictiva y un $Q^{2}$ menor que 0 indica que el modelo carece de relevancia predictiva. La tabla 9 muestra los valores de la varianza explicada y los valores de la prueba de Blindfolding a través de valida-

Tabla 9. Efectos sobre variables endógenas y exógenas

\begin{tabular}{|c|c|c|c|c|c|}
\hline Hipótesis & $\mathbf{R}^{2}$ & $Q^{2}$ & Efecto & Correlación & Varianza explicada \\
\hline Utilidad percibida (PU) & 0,6584 & 0,4942 & & & \\
\hline $\mathrm{H} 2.2: \mathrm{PeU} \rightarrow \mathrm{PU}$ & & & 0,1215 & 0,4565 & $5,55 \%$ \\
\hline $\mathrm{H} 3.1: \mathrm{JR} \rightarrow \mathrm{PU}$ & & & 0,5575 & 0,7764 & $43,28 \%$ \\
\hline H4.1: CSE $\rightarrow$ PU & & & 0,0137 & 0,3758 & $0,51 \%$ \\
\hline $\mathrm{H} 5.1: \mathrm{UA} \rightarrow \mathrm{PU}$ & & & 0,1338 & 0,6123 & $8,19 \%$ \\
\hline H6.1: TS $\rightarrow$ PU & & & 0,1444 & 0,5746 & $8,30 \%$ \\
\hline Facilidad de uso percibida (PeU) & 0,3675 & 0,3028 & & & \\
\hline $\mathrm{H} 5.2: \mathrm{UA} \rightarrow \mathrm{PeU}$ & & & 0,3887 & 0,5401 & $20,99 \%$ \\
\hline $\mathrm{H} 4.2: \mathrm{CSE} \rightarrow \mathrm{PeU}$ & & & 0,3126 & 0,4977 & $15,56 \%$ \\
\hline $\mathrm{H} 6.2: \mathrm{TS} \rightarrow \mathrm{PeU}$ & & & 0,0078 & 0,2612 & $0,20 \%$ \\
\hline Intención de uso (BI) & 0,5123 & 0,424 & & & \\
\hline $\mathrm{H} 1.1: \mathrm{PU} \rightarrow \mathrm{BI}$ & & & 0,4235 & 0,6641 & $28,12 \%$ \\
\hline $\mathrm{H} 2.1: \mathrm{PeU} \rightarrow \mathrm{BI}$ & & & 0,2631 & 0,5276 & $13,88 \%$ \\
\hline H7.1: PEN $\rightarrow$ BI & & & 0,1498 & 0,6158 & $9,22 \%$ \\
\hline
\end{tabular}


ción redundante $Q^{2}$, calculados mediante el software estadístico SmartPLS. Se obtuvieron los resultados siguientes: «utilidad percibida» (PU = 0,4942), «facilidad de uso percibida» ( $\mathrm{PeU}=0,3028)$ e «intención de uso» $(\mathrm{BI}=0,424)$, por lo que, según el criterio descrito anteriormente, se argumenta que el modelo de investigación propuesto tiene una relevancia predictiva.

\section{ANÁLISIS DE RESULTADOS}

En nuestra investigación, el TAM fue adaptado para predecir la aceptación tecnológica de los sistemas de formación en línea con la finalidad de determinar las variables claves en el desarrollo de cualquier proyecto estratégico de m-learning. Observamos que es importante conocer qué factores describen la aceptación de la tecnología de los usuarios. Estas dimensiones tendrían impactos importantes en los procesos de innovación, negocio y marketing dentro de las instituciones educativas. Los constructos del TAM que han sido utilizados en esta investigación explican el uso del $m$-learning para los smartphones. Encontramos algunas excepciones: la variable "autocapacidad informática» (CSE) no tiene un efecto importante en el constructo «utilidad percibida» $(\mathrm{PU})$ y la variable «soporte técnico» (TS) no tiene ningún efecto en el constructo «facilidad de uso percibida» (PeU). Como se podría predecir desde el principio, los valores alfa de Cronbach en el constructo "autocapacidad informática» (CSE) mostraron que los usuarios de m-learning consideran que sus conocimientos en el uso de su smartphone son altos. Debido a esta razón, para nuestro modelo, la variable «autocapacidad informática» (CSE) no se considera como un impedimento para utilizar sistemas de m-learning. Esto también puede deberse a la brecha generacional millennial. Los jóvenes tienen esa capacidad de adaptarse a las tecnologías con gran facilidad, sin necesidad de emplear un «soporte técnico» (TS) como en épocas anteriores. Nuestra conclusión es que las habilidades de los estudiantes sobre el uso de cualquier tecnología tienen un impacto positivo en la «facilidad de uso percibida» (PeU), mientras que la «percepción de utilidad» (PU) es un factor altamente considerable para la incorporación de los sistemas de formación online.

En el modelo propuesto en este estudio incluimos dos variables: "Soporte técnico» (TS) y "gozo percibido» (PEN). Se encontró que el «soporte técnico» (TS) tiene un impacto positivo en la «utilidad percibida» (PU) y en la «facilidad de uso percibida» (PeU), sin embargo, este factor no es significativo estadísticamente en nuestro análisis. La razón es que los estudiantes ya saben cómo utilizar sus dispositivos móviles y saben muy bien cómo acceder a los sistemas de formación en línea. Por este motivo, el «soporte técnico» (TS) no tiene influencia sobre la «facilidad de uso percibida» (PeU). Observamos que los estudiantes perciben que el apoyo técnico es útil bajo ciertas circunstancias. Lo anterior también refuerza nuestra conclusión de la brecha generacional. En la actualidad, los jóvenes poseen una facilidad casi innata para la tecnología. Además, hoy en día, las interfaces de los sistemas les resultan muy intuitivas.

En relación con la variable «gozo percibido» (PEN), los resultados muestran la existencia de una relación positiva con el uso del smartphone para acceder al sistema de formación en línea; sin embargo, no tiene una relevancia estadística. Esto podría deberse, principalmente, a que los estudiantes no se sienten del todo cómodos cuando utilizan su smartphone para realizar algunas actividades dentro del sistema. Concluimos que esta variable tiene una implicación directa en el ámbito de negocio en las universidades. Debido a que el proceso de enseñanza también requiere de un proceso de negocio en el que debe existir un oferente y un ofertante, las instituciones han de valorar esta situación y ofrecer la comodidad a sus ofertantes dentro de sus cursos en línea mediante aplicaciones más atractivas, actividades dinámicas etc., que conduzcan a una mejora en el proceso de aprendizaje y, por ende, a la lealtad del estudiante como cliente. La variable «relevancia en el trabajo» (JR) tiene un impacto positivo en la «utilidad percibida» (PU) y, al mismo tiempo, encontramos un alto significado estadístico. Se puede suponer que los usuarios de los sistemas de formación en línea consideran el uso del smartphone como una herramienta útil en su proceso de aprendizaje. Sin embargo, como se observa en el "gozo percibido» (PEN), para llevar a cabo un análisis más preciso, debe considerarse el tipo de actividad realizada.

Encontramos que la relación estadísticamente más significativa es la existente entre la «adaptación del usuario» (UA) y la «facilidad de uso percibida» (PeU). Podría indicar que los usuarios de los sistemas de $m$-learning no tienen ninguna dificultad para trabajar desde su smartphone. Esto es debido a que los usuarios están muy adaptados a sus dispositivos. Además de ello, la relación entre la «adaptación del usuario» (UA) 
y la «utilidad percibida» (PU) tiene una influencia positiva, pero es significativamente menor, por lo que podemos afirmar que los estudiantes perciben que el uso del sistema de formación en línea está bien adaptado para el smartphone. A pesar de ello, hacemos nuevamente énfasis en que estas variables dependen en gran medida del tipo de actividad realizada (lectura, escritura, observación, escucha, etc.), así como en el «gozo percibido» (PEN) y en la «relevancia del trabajo» (JR).
Respecto a las contribuciones a otras áreas, como la de innovación educativa, negocios y marketing educativo, concluimos que la aceptación dependerá significativamente del tipo de actividad que el estudiante vaya a realizar a través de su dispositivo móvil, por lo que sugerimos que el desarrollo e implementación de una plataforma de m-learning debe ser cuidadosamente elaborada de acuerdo al tipo de tarea que se llevará a cabo en cada dispositivo.

\section{CONCLUSIONES E IMPLICACIONES PRÁCTICAS}

A continuación, presentamos de forma resumida las principales conclusiones e implicaciones prácticas para la formulación de estrategias de m-learning teniendo en cuenta la aceptación tecnológica:

- Los estudiantes dominan el uso de los dispositivos móviles, por lo que no necesitan formación, ni soporte técnico para su utilización. Por lo tanto, la formación de usuarios y el soporte técnico no serán variables clave del desarrollo estratégico de proyectos de m-learning.

- Los estudiantes consideran útil el empleo del smartphone en su proceso de aprendizaje. Por ello, a partir de los resultados obtenidos en este estudio, sugerimos que todo proyecto estratégico de m-learning debería tener en cuenta que el estudiante se sienta cómodo cuando realice sus actividades de aprendizaje mediante la creación y adaptación de contenidos al dispositivo móvil. Con ello se fortalecería el binomio enseñanza-aprendizaje.

- En el desarrollo de un proyecto integral de m-learning es necesario considerar la relevancia que tendrán las actividades educativas y cómo estas, en conjunto con las tecnologías, deben adaptarse a las necesidades de los estudiantes. Un factor fundamental durante el desarrollo de proyectos de m-learning es la elaboración de estrategias educativas que integren las tecnologías móviles como medio de soporte para la generación y la proliferación de conocimiento. Estas estrategias deben tener en cuenta que todas las actividades que se oferten en los cursos académicos sean importantes para el aprendizaje y que, además, se adapten perfectamente a los dispositivos móviles.

- Los estudiantes consideran que los dispositivos móviles son útiles para realizar sus actividades académicas. Por esta razón, a pesar de las implicaciones que hemos comentado anteriormente, los desarrolladores de proyectos de m-learning en las instituciones educativas deben fomentar la aplicación de los dispositivos móviles en las actividades implícitas en las asignaturas y, a su vez, deben considerar que estas actividades sean intuitivas y que los estudiantes las perciban como un elemento útil dentro de su proceso de formación.

Cheung, R. y Vogel, D. (2013). Predicting user acceptance of collaborative technologies: an extension of the technology acceptance model for e-learning. Computers \& Education, 63, 160-175. doi: 10.1016/j.compedu.2012.12.003.

Chow, M., Herold, D. K., Choo, T.-M. y Chan, K. (2012). Extending the technology acceptance model to explore the intention to use Second Life for enhancing healthcare education. Computers \& Education, 59(4), 1.136-1.144. doi: 10.1016/j.compedu.2012. 05.011. doi: 10.1016/j.csi.2011.01.003. 
Davis, F. D. (1985). A Technology Acceptance Model for Epirically Testing New End-User Information Systems: Theory and Results. Massachusetts Institute of Technology.

Davis, F. D., Bagozzi, R. P. y Warshaw, P. R. (1989). User acceptance of computer technology: a comparison of two theoretical models. Management Science, 35(8), 982-1.003. doi: $10.1287 / \mathrm{mnsc} .35 .8 .982$.

Davis, F. D., Bagozzi, R. P. y Warshaw, P. R. (1992). Extrinsic and intrinsic motivation to use computers in the workplace. Journal of Applied Social Psychology, 22(14), 1.111-1.132.

Deng, L. y Tavares, N. J. (2013). From Moodle to Facebook: exploring students' motivation and experiences in online communities. Computers \& Education, 68, 167-176. doi: 10.1016/j.compedu.2013.04.028.

Fishbein, M. y Ajzen, I. (1975). Belief, Attitude, Intention, and Behavior: An Introduction to Theory and Research. Addison-Wesley.

Govindarajan, V. (2012). Developing countries are revolutionizing mobile banking. Harvard Business Review, 1-2.

Hair, J. F., Ringle, C. M. y Sarstedt, M. (2011). PLS-SEM: indeed a silver bullet. The Journal of Marketing Theory and Practice, 19(2), 139-152. doi: 10.2753/MTP10696679190202.

Hair, J. F., Sarstedt, M., Ringle, C. M. y Mena, J. A. (2011). An assessment of the use of partial least squares structural equation modeling in marketing research. Journal of the Academy of Marketing Science, 40(3), 414-433. doi: 10.1007/s11747-011-0261-6.

Henseler, J., Dijkstra, T. K., Sarstedt, M., Ringle, C. M., Diamantopoulos, A., Straub, D. W., ... Calantone, R. J. (2014). Common beliefs and reality about PLS: Comments on Ronkko and Evermann (2013). Organizational Research Methods, 17(2), 182-209. doi: 10.1177/1094428114526928.

Holden, H. y Rada, R. (2011). Understanding the influence of perceived usability and technology self-efficacy on teachers' technology acceptance. Journal of Research on Technology in Education, 43(4), 343-367.
Kim, S. H. (2008). Moderating effects of job relevance and experience on mobile wireless technology acceptance: adoption of a smartphone by individuals. Information \& Management, 45(6), 387-393. doi: 10.1016/j.im.2008.05.002.

Martínez-Torres, M. R., Toral Marín, S. L., Barrero García, F., Gallardo Vázquez, S., Arias Oliva, M. y Torres, T. (2008). A technological acceptance of e-learning tools used in practical and laboratory teaching, according to the European higher education area. Behaviour \& Information Technology, 27(6), 495505. doi: 10.1080/01449290600958965.

Ngai, E. W. T., Poon, J. K. L. y Chan, Y. H. C. (2007). Empirical examination of the adoption of WebCT using TAM. Computers \& Education, 48(2), 250-267. doi: 10.1016/j.compedu.2004.11.007.

Roldán, J. L. y Sánchez-Franco, M. J. (2012). Variance-based structural equation modeling: guidelines for using partial least squares in information systems research. En M. S. Mora, M. Gelman, O. Steenkamp y A. Raisinghani (eds.), Research Methodologies, Innovations and Philosophies in Software Systems Engineering and Information Systems (p. 221). Hershey PA: IGI Global.

Terzis, V. y Economides, A. A. (2011). The acceptance and use of computer based assessment. Computers \& Education, 56(4), 1.032-1.044. doi: 10.1016/j.compedu.2010.11.017.

Venkatesh, V. y Bala, H. (2008). Technology acceptance model 3 and a research agenda on interventions. Decision Sciences, 39(2), 273-315. doi: 10.1111/j.1540-5915.2008.00192.x.

Venkatesh, V. y Davis, F. D. (2000). A theoretical extension of the technology acceptance model: four longitudinal field studies. Management Science, 46(2), 186-204.

Venkatesh, V., Morris, M. G., Davis, G. B. y Davis, F. D. (2003). User acceptance of information technology: toward a unified view. MIS Quarterly, 27(3), 425-478.

Yu, J., Ha, I., Choi, M. y Rho, J. (2005). Extending the TAM for a t-commerce. Information \& Management, 42(7), 965-976. doi: 10.1016/j.im.2004.11.001. 
Este máster oficial [60 créditos ECTS] se inicia en octubre y febrero de cada año y su duración normal es de 12 meses.

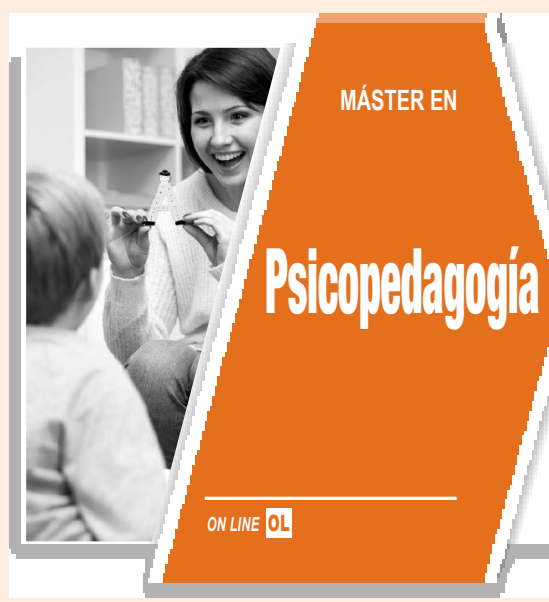

DIRIGIDO A: Personas vinculadas con el mundo de la educación formal y no formal que deseen actualizar su formación. El estudiante de este máster ha de estar interesado por la labor del educador en un enfoque educativo inclusivo en el ámbito de la educación formal, y en el trabajo con diferentes grupos o colectivos sociales, favoreciendo la mejora de sus condiciones de vida y la disminución de las desigualdades por motivos de carácter social y cultural.

OBJETIVOS: Permite el desempeño de una labor profesional especializada, avanzada y focalizada en el análisis, la planificación y la intervención para la mejora de los contextos educativos, sociolaborales y sociocomunitarios, de ahí la necesidad de una formación de posgrado que permita el desarrollo de las competencias específicas y multidisciplinares requeridas para su práctica profesional.

Este máster oficial en Formación del Profesorado de Educación Secundaria Obligatoria, Bachillerato, Formación Profesional y Enseñanza de Idiomas [60 créditos ECTS] se inicia en octubre y febrero de cada año y su duración normal es de 12 meses.

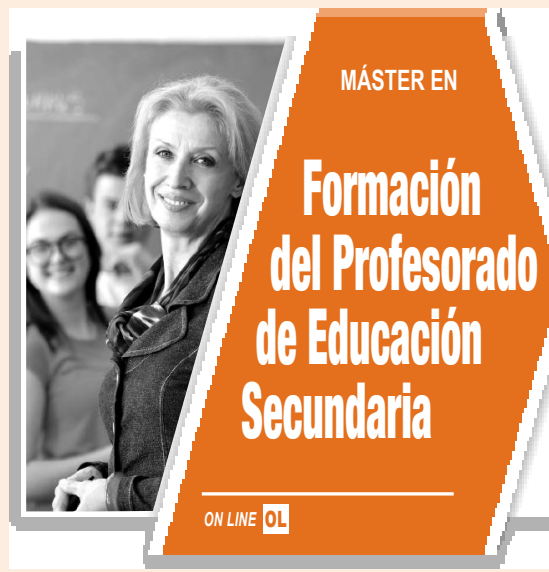

DIRIGIDO A: La universalización de la enseñanza secundaria y el incremento de la atención a la diversidad de alumnos en todos los niveles de enseñanza ha hecho más patente la necesidad de mayor formación didáctica. El educador ya no solo debe ser un experto en su materia, sino que debe tener la suficiente capacidad didáctica para adaptar la misma a grupos de alumnos muy heterogéneos en intereses, capacidades y actitudes.

OBJETIVOS: Adquirir todas las habilidades y competencias necesarias para poder desarrollar una carrera profesional en el ámbito de la enseñanza en los niveles de Educación Secundaria Obligatoria y Bachillerato, Formación Profesional y Enseñanzas de Idiomas, ya sea para dar clase en centros públicos, privados o concertados. 


\section{Psicología (Rama CC. de la Salud)}

Siguiendo el modelo científico-profesional de psicólogo (o scientistpractitioner), se trata de aportar a los alumnos los conocimientos científicos necesarios para comprender, interpretar, analizar y explicar el comportamiento humano, así como para evaluar e intervenir en el ámbito individual y social, con el fin de que los psicólogos y la psicología promuevan y mejoren la salud y la calidad de vida de las personas.

PLAN DE ESTUDIOS (la obtención del grado conlleva la realización de 240 créditos)

\begin{tabular}{|c|c|c|c|}
\hline CURSO & ASIGNATURAS & TIPO & CRÉD \\
\hline 1 & Historia de la Psicología & $\mathrm{T}$ & 6 \\
\hline 1 & Psicología del Aprendizaje & $\mathrm{T}$ & 6 \\
\hline 1 & 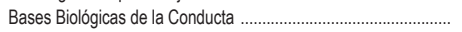 & $\mathrm{T}$ & 6 \\
\hline 1 & Tecnologías de la Información y de la Comunicación ..................... & $\mathrm{T}$ & 6 \\
\hline 1 & Sociología General ................................................................ & T & 6 \\
\hline 1 & 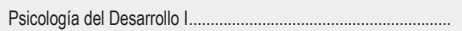 & $\mathrm{T}$ & 6 \\
\hline 1 & Introducción a la Antropologia & $\mathrm{T}$ & 6 \\
\hline 1 & Métodos, Diseños y Técnicas de Investigación Psicológica ......... & $\mathrm{T}$ & 6 \\
\hline 1 & Psicologia Social & $\mathrm{T}$ & 6 \\
\hline 1 & 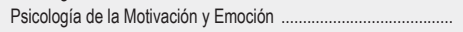 & T & 6 \\
\hline 2 & Neurociencia & B & 6 \\
\hline 2 & 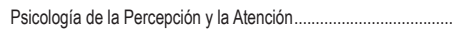 & B & 6 \\
\hline 2 & 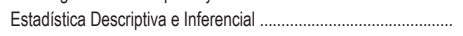 & B & 6 \\
\hline 2 & 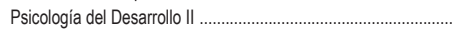 & B & 6 \\
\hline 2 & Psicologia de la Personalidad y las Diferencias Individuales ....... & B & 6 \\
\hline 2 & 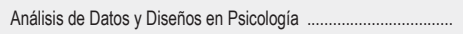 & B & 6 \\
\hline 2 & Psicologia de la Memoria & B & 6 \\
\hline 2 & Evaluación Psicológica & B & 6 \\
\hline 2 & Psicologia de los Grupos & B & 6 \\
\hline 2 & 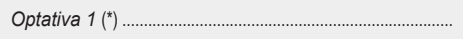 & 0 & 6 \\
\hline 3 & 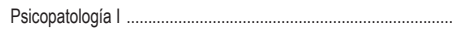 & B & 6 \\
\hline 3 & 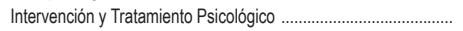 & B & 6 \\
\hline 3 & Psicometría & B & 6 \\
\hline 3 & 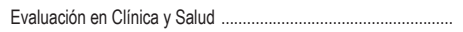 & B & 6 \\
\hline 3 & Optativa $2\left(^{*}\right)$ & 0 & 6 \\
\hline 3 & 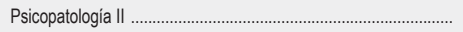 & B & 6 \\
\hline 3 & 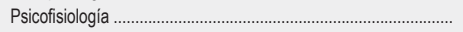 & B & 6 \\
\hline 3 & 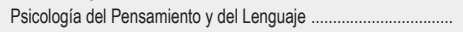 & B & 6 \\
\hline 3 & 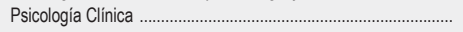 & B & 6 \\
\hline 3 & 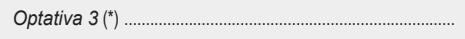 & 0 & 6 \\
\hline 4 & Psicología de la Salud & B & 6 \\
\hline 4 & Psicologia de la Educación & B & 6 \\
\hline 4 & Psicología del Trabajo y de las Organizaciones ................................ & B & 6 \\
\hline 4 & 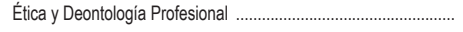 & $\mathrm{B}$ & 6 \\
\hline 4 & 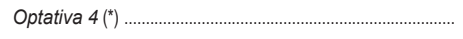 & 0 & 6 \\
\hline 4 & Intervención Psicosocial ............................................................. & B & 6 \\
\hline 4 & Optativa $5\left(^{*}\right)$ & 0 & 6 \\
\hline 4 & PRÁCTICUM & B & 9 \\
\hline 4 & TRABAJO FIN DE GRADO & B & 9 \\
\hline
\end{tabular}

\section{Historia}

Se conjugan los conocimientos humanísticos básicos y generalistas con el aprendizaje de las herramientas y técnicas de las nuevas TIC. Los estudiantes adquiriran la formación, los conocimientos y las habilidades necesarias para permitirles el pleno desarrollo de las funciones relacionadas con la investigación y la enseñanza de la historia, para que comprendan y hagan comprensibles a los demás los acontecimientos del pasado.

PLAN DE ESTUDIOS (la obtención del grado conlleva la realización de 240 créditos)

\begin{tabular}{|c|c|c|c|}
\hline CURSO & ASIGNATURAS & TIPO & CRÉD \\
\hline 1 & Ciencia Histórica. Conceptos y Etapas de la Historia Universal .. & $\mathrm{T}$ & 6 \\
\hline 1 & Geografia General . & $\mathrm{T}$ & 6 \\
\hline 1 & 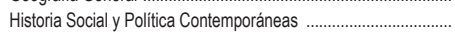 & $\mathrm{T}$ & 6 \\
\hline 1 & Tecnologia y Gestión de la Información y del Conocimiento ......... & T & 6 \\
\hline 1 & 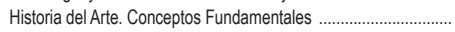 & $\mathrm{T}$ & 6 \\
\hline 1 & Antropologia General & $\mathrm{T}$ & 6 \\
\hline 1 & España Actual & $\mathrm{T}$ & 6 \\
\hline 1 & 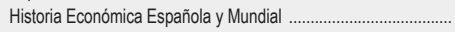 & $\mathrm{T}$ & 6 \\
\hline 1 & Historia de América & $\mathrm{T}$ & 6 \\
\hline 1 & Prehistoria Universal & $\mathrm{T}$ & 6 \\
\hline 2 & 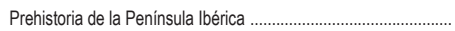 & B & 6 \\
\hline 2 & 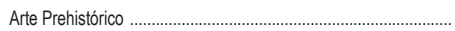 & B & 6 \\
\hline 2 & Historia del Mundo Grecorromano & B & 6 \\
\hline 2 & 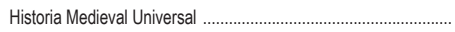 & B & 6 \\
\hline 2 & Historia de España Antigua & B & 6 \\
\hline 2 & Historia de la Cultura Escrita & B & 6 \\
\hline 2 & 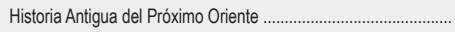 & B & 6 \\
\hline 2 & Pensamiento Antiguo y Medieval ................................................... & B & 6 \\
\hline 2 & Historia de las Sociedades Peninsulares en la Edad Media ......... & B & 6 \\
\hline 2 & Optativa $1\left(^{*}\right)$ & 0 & 6 \\
\hline 3 & Historia del Arte Antiguo y Medieval .. & B & 6 \\
\hline 3 & 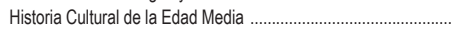 & B & 6 \\
\hline 3 & 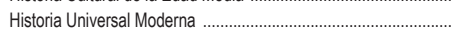 & B & 6 \\
\hline 3 & 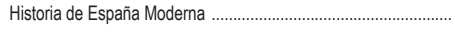 & B & 6 \\
\hline 3 & Optativa $2\left(^{*}\right)$... & 0 & 6 \\
\hline 3 & Pensamiento Moderno & B & 6 \\
\hline 3 & Historia Universal Contemporánea & B & 6 \\
\hline 3 & Fundamentos de Arqueologia & B & 6 \\
\hline 3 & 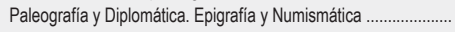 & B & 6 \\
\hline 3 & Optativa $3\left(^{*}\right)$. & 0 & 6 \\
\hline 4 & 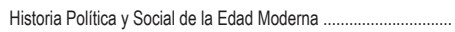 & B & 6 \\
\hline 4 & Historia Contemporánea de España. El Siglo XIX ....................... & B & 6 \\
\hline 4 & Pensamiento Contemporáneo & B & 6 \\
\hline 4 & 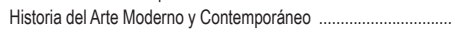 & B & 6 \\
\hline 4 & 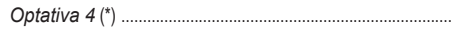 & 0 & 6 \\
\hline 4 & 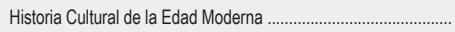 & B & 6 \\
\hline 4 & 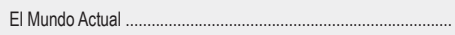 & B & 6 \\
\hline 4 & $\begin{array}{l}\text { Métodos y Técnicas de Investigación Histórica. Tendencias His- } \\
\text { toriográficas Actuales }\end{array}$ & B & 6 \\
\hline 4 & TRABAJO FIN DE GRADO & B & 12 \\
\hline
\end{tabular}

${ }^{*}$ ) La lista de asignaturas optativas se puede consultar en www.udima.es. $\quad \mathrm{T}=$ Formación básica; $\mathrm{B}=$ Formación obligatoria; $\mathrm{O}=$ Asignatura optativa 